Pacific

Journal of

Mathematics

\title{
AREA-MINIMIZING MINIMAL GRAPHS OVER NONCONVEX DOMAINS
}

Michael Dorff, Denise Halverson, and Gary Lawlor 


\title{
AREA-MINIMIZING MINIMAL GRAPHS OVER NONCONVEX DOMAINS
}

\author{
Michael Dorff, Denise Halverson, and Gary Lawlor
}

\begin{abstract}
Sufficient conditions for which a minimal graph over a nonconvex domain is area-minimizing are presented. The conditions are shown to hold for subsurfaces of Enneper's surface, the singly periodic Scherk surface, and the associated surfaces of the doubly periodic Scherk surface which previously were unknown to be area-minimizing. In particular these surfaces are graphs over (angularly accessible) domains which have a nice complementary set of rays. A computer assisted method for proving polynomial inequalities with rational coefficients is also presented. This method is then applied to prove more general inequalities.
\end{abstract}

\section{Introduction.}

In this paper, we establish conditions under which a minimal graph over a certain type of nonconvex domain is area-minimizing. In particular, we consider those domains that have a "nice complementary set of rays". Loosely speaking, a closed domain $D$ with a piecewise smooth boundary has a nice complementary set of rays if its complement can be written as the union of non-intersecting open rays emanating from $\partial D$ that are non-tangent to $\partial D$. It is shown that the compact closure of a bounded domain $D$ with connected smooth boundary has a nice complementary set of rays if and only if $D$ is angularly accessible. Such domains are a subclass of the linearly accessible domains. Linearly accessible domains are in turn associated with the close-to-convex functions, which have been studied from a geometric function theory viewpoint both in the analytic (see [5]) and the harmonic (see [1]) cases. We will show that certain regions of Enneper's surface, the singly periodic Scherk surface, and the associated surfaces of the doubly periodic Scherk surface are area-minimizing. Furthermore, in order to prove certain inequalities necessary for our results, a computer assisted strategy is introduced. The strategy is designed to prove inequalities involving polynomials with rational coefficients. Although the required inequalities are not initially of this form, they can be modified to obtain inequalities of this form. 


\section{Preliminaries.}

Let $M$ be an orientable surface that arises from a differentiable mapping $X$ from a domain $\Omega \subset \mathbb{R}^{2}$ into $\mathbb{R}^{3}$, so that $X(u, v)=(x(u, v), y(u, v), z(u, v))$. Fix a point $P$ on $M$. Let $\mathbf{t}$ denote a vector tangent to $M$ at $P$ and $\mathbf{n}$ the unit normal vector to $M$ at $P$. Then $\mathbf{t}$ and $\mathbf{n}$ determine a plane that intersects $M$ in a curve $\beta$. The normal curvature $\kappa_{\mathbf{t}}$ at $P$ is defined to have the same magnitude as the curvature of $\beta$ at $P$ with the sign of $\kappa_{\mathbf{t}}$ chosen to be consistent with the choice of orientation of $M$. The principal curvatures, $\kappa_{1}$ and $\kappa_{2}$, of $M$ at $P$ are the maximum and minimum of the normal curvatures $\kappa_{\mathbf{t}}$ as $\mathbf{t}$ ranges over all directions in the tangent space. The mean curvature of $M$ at $P$ is the average value $H=\frac{1}{2}\left(\kappa_{1}+\kappa_{2}\right)$.

\section{Definition 2.1.}

(a) A minimal surface in $\mathbb{R}^{3}$ is a regular surface for which the mean curvature is zero at every point.

(b) A surface of finite area is area-minimizing if it has the least surface area of any surface having that particular boundary. A surface of infinite area is area-minimizing if each of its compact subsurfaces is area-minimizing.

Every area-minimizing surface is a minimal surface, but the converse is not true. In fact, it is difficult to prove that a specific surface is areaminimizing. However, there is the following classical result [see [6], 5.4.18 or see [11], 6.1]:

Theorem 2.2. A minimal graph over a convex domain is area-minimizing.

It follows that any subsurface of a minimal graph over a convex domain is also area-minimizing. In contrast, minimal surfaces containing areaminimizing pieces may not be area-minimizing. For example, consider the minimal surface given by

$$
X(u, v)=\left(u-u^{3} / 3+u v^{2}, v-v^{3} / 3+v u^{2}, u^{2}-v^{2}\right)
$$

known as Enneper's surface. Let $U_{r}$ be the disc of radius $r$ in the $u, v$ plane centered at the origin. Let $\pi_{r}$ denote the projection of $X\left(U_{r}\right)$ to the $x, y$-plane. Then $X\left(U_{r}\right)$ is a graph over $\pi_{r}$ for $r \leq 1$. Although $\pi_{1}$ is not convex, the convex hull of $\pi_{r}$ is contained in $\pi_{1}$ precisely for $r \leq r_{0}=$ $\sqrt{2^{2 / 3}-1} \approx 0.766$ and hence any subsurface of $X\left(U_{r_{0}}\right)$ is area-minimizing. For $r_{0}<r \leq 1$, the convex hull of $\pi_{r}$ is no longer contained in $\pi_{1}$ and it has been unknown whether or not $X\left(U_{r}\right)$ is area-minimizing for any of these $r$ values. However, it is known for $r \leq 1$ that $X\left(U_{r}\right)$ has least area among topological disks with the same boundary [14]. For $1<r<\sqrt{3}, X\left(U_{r}\right)$ still has no self intersections, but is no longer a graph and does not minimize area [12]. Oprea ([13]) shows that this follows from a theorem of Schwarz, 
since the image of the Gauss map then contains an entire hemisphere in its interior.

The fact that a minimal graph over a nonconvex domain need not minimize area is illustrated in [11].

The types of surfaces to which we will apply our results are those that have domains that satisfy the following definition:

Definition 2.3. Let $D$ be a closed region in $\mathbb{R}^{2}$ with piecewise smooth boundary. Suppose that $\Upsilon$ is a set of rays having the following properties:

(1) $\mathcal{R} \cap D=\partial \mathcal{R}$ for every $\mathcal{R} \in \Upsilon$.

(2) $\mathcal{R} \cap \mathcal{R}^{\prime} \subset \partial D$ for every distinct pair of rays $\mathcal{R}, \mathcal{R}^{\prime} \in \Upsilon$.

(3) $\overline{D^{C}}=\bigcup_{\mathcal{R} \in \Upsilon} \mathcal{R}$.

(4) There is a $\delta>0$ and a set $A \subset \partial D$ so that the one-dimensional Hausdorff measure $\mathcal{H}^{1}(A)$ equals 0 and for all $p \in \partial D-A$, the angle between $\partial D$ and any ray $\mathcal{R} \in \Upsilon$ emanating from $p$ is defined and is at least $\delta$.

Then $\Upsilon$ is called a nice complementary set of rays for $D$ in $\mathbb{R}^{2}$. If $\Upsilon$ satisfies (1)-(3), then $\Upsilon$ is called a complementary set of rays for $D$ in $\mathbb{R}^{2}$. The points $p \in \partial D$ at which $\partial D$ is smooth and which have a unique $\mathcal{R}(p) \in \Upsilon$ radiating from them are called the standard points of $\partial D$.

Note that it follows from Conditions (1) and (3) that for every $p \in \partial D$ there is at least one $\mathcal{R} \in \Upsilon$ such that $\partial \mathcal{R}=p$. Furthermore, the nonstandard points of $\partial D$ are countable. The rays radiating from a nonstandard point on the smooth parts of $\partial D$ form an angle of positive measure. If the non-standard points were uncountable, then $\mathbb{R}^{2}$ would contain an uncountable collection of pairwise disjoint open sets, which is not possible.

Lemma 2.4. Suppose that $D$ is a compact domain with piecewise smooth connected boundary and that $\Upsilon$ is a nice complementary set of rays for $D$. Then no ray in $\Upsilon$ is contained in the tangent cone of any point of $\partial D$.

Proof. Let $B$ be a ball in $\mathbb{R}^{2}$ that contains $D$ in its interior. Note that there is a 1-1 correspondence between the rays of $\Upsilon$ and the points of $\partial B$. Let $\psi: \partial B \rightarrow \partial D$ be the map projecting $\partial B$ onto $\partial D$ via the rays of $\Upsilon$. It can be shown from the properties of a nice complementary set of rays that $\psi$ is an order preserving surjection in the sense that whenever $a, b, c, d \in \partial D$ so that $\{a, b\}$ separates $c$ and $d$, then either $\{\psi(a), \psi(b)\} \cap\{\psi(c), \psi(d)\} \neq \emptyset$ or $\{\psi(a), \psi(b)\}$ separates $\psi(c)$ and $\psi(d)$. It follows that $\psi$ is continuous and that each point preimage is a point or a connected arc.

Let $J_{1}, J_{2}, \ldots, J_{n}$ be smooth arcs forming $\partial D$. Define $a_{n}=J_{1} \cap J_{n}$ and $a_{i}=J_{i} \cap J_{i+1}$ for $i=1, \ldots, n-1$. Then the endpoints of $J_{i}$ are $a_{i-1}$ and $a_{i}$ for $i=2, \ldots n$ and the endpoints of $J_{1}$ are $a_{n}$ and $a_{1}$. If $\psi^{-1}\left(a_{i}\right)$ is a point, denote that point as $b_{i}$. If not, let $b_{i}$ be a point in the interior of the 
segment $\psi^{-1}\left(a_{i}\right)$. Let $K_{i}$ denote the arc in $\psi_{-1}\left(J_{i}\right)$ with endpoints $b_{i-1}$ and $b_{i}$ for $i=2, \ldots n$ or $b_{n}$ and $b_{1}$ if $i=n$.

It suffices to show that no ray in $\Upsilon$ meeting $K_{i}$ is contained in the tangent cone of a point of $J_{i}$. Suppose to the contrary that $\mathcal{R} \in \Upsilon$ meets $K_{i}$ at $q$ and is contained in the tangent cone to $J_{i}$ at $p=\partial \mathcal{R}$. Clearly, $q$ can not be contained in the interior of $\psi^{-1}(p)$. Hence given any set $A \subset J_{i}$ of Hausdorff measure 0 , there is a sequence of points $\left\{q_{i}\right\} \subset \psi^{-1}\left(K_{i}-(A \cup\{p\})\right)$ so that $q_{i} \rightarrow q$.

Let $\mathbf{N}(s)$ denote the outward unit normal to $J_{i}$ at $s$. It follows from the smoothness of $J_{i}$ that $\mathbf{N}(s)$ is continuous. Let $\mathbf{R}(t)$ denote the unit vector in the direction of the unique ray of $\Upsilon$ which meets $K$ at $t$. Then $\mathbf{R}(t)=\frac{t-\psi(t)}{\|t-\psi(t)\|}$ is also continuous. Therefore $\mathbf{N}(\psi(t)) \cdot \mathbf{R}(t)$ is continuous on $K_{i}$. Hence $\mathbf{N}\left(\psi\left(q_{i}\right)\right) \cdot \mathbf{R}\left(q_{i}\right) \rightarrow 0$. This contradicts (4) of the definition of a nice complementary set of rays. Therefore, no ray in $\Upsilon$ is contained in the tangent cone of any point of $\partial D$.

Recall that a region is linearly accessible if its complement can be written as the union of non-crossing rays. The rays are non-crossing in the sense that they may only meet at their endpoints. Furthermore, a region is said to be angularly accessible of order $\beta, \beta \in[0,1]$, if its complement can be written as the union of non-crossing rays so that each ray is the bisector of a sector of measure $(1-\beta) \pi$ that is contained in the complement of the region. Thus any angularly accessible region is linearly accessible. A linearly accessible region that is not angularly accessible of order $\beta<1$, is said to be strictly linearly accessible.

We will say that a closed domain $D$ is angularly (or linearly) accessible if its interior is angularly (or linearly) accessible. Given an angularly accessible domain $D$ of order $\beta$, a set of rays satisfying the definition is called an access set of rays of order $\beta$ for $D$.

Clearly the concepts of a domain being strictly angularly accessible versus having a nice complementary set of rays are related. A priori, however, it is not obvious that either condition implies the other, since:

1) A measure zero set of rays is excluded from the angle requirement in Definition 2.3.

2) The angle requirement in Definition 2.3 is local, while the requirement that a wedge miss an entire region is global.

3) Angular accessibility does not require rays to have their endpoints on the boundary of the domain; there could be "feathers" where a ray $\mathcal{R}$ extending from $D$ is not a ray of the access set, but some of the rays of the access set extend out from $\mathcal{R}$.

The following theorem, however, reconciles these differences for piecewise smooth domains: 
Theorem 2.5. A compact domain $D$ with piecewise smooth connected boundary has a nice complementary set of rays if and only if $D$ is angularly accessible of order $\beta<1$.

Proof. Suppose that $D$ has a nice complementary set of rays, $\Upsilon$. Clearly, $D$ is linearly accessible. Assume that $D$ is strictly linearly accessible. As in Lemma 2.4 , let $B$ be a ball in $\mathbb{R}^{2}$ that contains $D$ and let $\psi: S \rightarrow \partial D$ be the map projecting $\partial D$ onto $\partial D$ via the rays of $\Upsilon$. As discussed in Lemma 2.4, for each $p \in \partial D, \psi^{-1}(p)$ is a point or a connected arc in $\partial D$.

Suppose there is a sequence $\left\{\mathcal{R}_{i}\right\} \subset \Upsilon$ so that the $\frac{1}{i}$-sector centered at $\mathcal{R}_{i}, S\left(\mathcal{R}_{i}, \frac{1}{i}\right)$, meets $D$ at more than one point. Without loss of generality assume that $\mathcal{R}_{i} \rightarrow \mathcal{R}$. Let $p_{i}=\partial \mathcal{R}_{i}$. Then $S\left(\mathcal{R}_{i}, \frac{1}{i}\right) \cap \partial D-p_{i} \neq \emptyset$. Choose $q_{i} \in S\left(\mathcal{R}_{i}, \frac{1}{i}\right) \cap \partial D-p_{i}$. Again, without loss of generality, we may assume that there is a smooth arc $J$ of $\partial D$ containing $\left\{p_{i}\right\}$ and $\left\{q_{i}\right\}$. Let $p=\partial \mathcal{R}$. Since $J \cap \mathcal{R}=p$, then $p_{i} \rightarrow p$ and $q_{i} \rightarrow p$. By the smoothness of $J$ there are points $r_{i} \in J$ between $q_{i}$ and $p_{i}$ so that the direction of the line tangent to $J$ at $r_{i}$ is $\frac{1}{i}$-close to the direction of $\mathcal{R}_{i}$. Since $r_{i} \rightarrow p$ it follows from the smoothness of $J$ that $\mathcal{R}$ is tangent to $J$. This is a contradiction to Lemma 2.4. Therefore $D$ is angularly accessible of order $\beta$ for some $\beta<1$.

Conversely, suppose that $D$ is angularly accessible of order $\beta<1$. Let $\Upsilon^{*}$ be an access set of rays of order $\beta$ for $D$. A nice complementary set of rays is obtained by first noting that for each point of $p \in \partial D$ there is a ray $\mathcal{R}_{p} \in \Upsilon$ such that $p=\partial \mathcal{R}_{p}$. Let $\mathcal{S}_{p}$ be the component of the complement of $\operatorname{int}(D) \cup\left(\bigcup\left\{\mathcal{R}_{q} \mid q \neq p\right\}\right)$ which contains $\mathcal{R}_{p}$. Let $\Gamma_{p}$ be the set of all rays emanating from $p$ into $\mathcal{S}_{p}$. Then the set $\Upsilon=\bigcup \Gamma_{p}$ is a nice complementary set of rays.

Lewandowski [10] has shown that an analytic function on the unit disc is close-to-convex if and only if its image domain is linearly accessible. There is a similar relationship between certain analytic functions and image domains that are angularly accessible of order $\beta<1[8]$.

\section{Results.}

As was previously mentioned, it is well-known that a minimal surface that is a graph over a convex domain has least area among all surfaces (graphs or not) having the same boundary. In this section we provide a new area minimization result for graphs over nonconvex domains. We begin by outlining a proof by calibrations for the convex domain case (found in [11]).

Theorem 3.1. Let $D$ be a convex domain in $\mathbb{R}^{2}$. Let $f(x, y)$ be a function defined on $D$ whose graph $M$ is a regular minimal surface in $\mathbb{R}^{3}$. Then $M$ has least area among all surfaces having the same boundary as $M$.

Proof. Let $C$ be the vertical cylinder over $D$. Define a differential 2 -form $\phi$ at points $(x, y, f(x, y)) \in M$ by letting it be the unit length dual of the 
tangent plane, given by

$$
\phi=\frac{1}{\sqrt{1+f_{x}^{2}+f_{y}^{2}}}\left(-f_{x} d y d z-f_{y} d z d x+d x d y\right) .
$$

Extend $\phi$ to all of $C$ by letting it be constant in the vertical direction: $\phi(x, y, z)=\phi(x, y, f(x, y))$. A straightforward calculation shows that a form $\phi$ defined in this way is closed if and only if $M$ is a minimal surface.

Now let $S$ be any other surface whose boundary is the same as that of $M$. If $S$ lies entirely in $C$, so that $\phi$ is defined on $S$, then we have

$$
\operatorname{Area}(M)=\int_{M} \phi=\int_{S} \phi \leq \operatorname{Area}(S) .
$$

The middle equation is by Stokes' theorem, and the last inequality follows since $\phi$ returns a value less than or equal to 1 when applied to tangent planes of $S$.

If $S$ does not lie entirely in $C$, we employ the nearest point projection $\Pi$ onto $C$. Since $\Pi$ does not increase surface area, we have

$$
\operatorname{Area}(M)=\int_{M} \phi=\int_{\Pi(S)} \phi \leq \operatorname{Area}(\Pi(S)) \leq \operatorname{Area}(S) .
$$

Now if $D$ is a nonconvex set, then for any projection $\Pi$ onto $C$, the last inequality above may not hold. However, under certain circumstances we can omit Area $(\Pi(S))$ from the inequality string and still obtain

$$
\operatorname{Area}(M)=\int_{M} \phi=\int_{\Pi(S)} \phi \leq \operatorname{Area}(S) .
$$

This is because, although $\Pi(S)$ may have more area than $S$, the integration of $\phi$ may not be counting all the area of $\Pi(S)$.

Theorem 3.2. Let $D \subset \mathbb{R}^{2}$ be a closed region with a nice complementary set of rays $\Upsilon$. Let $M$ be a minimal surface in $\mathbb{R}^{3}$ which is a graph of a function $f(x, y)$ defined on $D$. For every standard $p \in \partial D$, suppose that

$$
|\mathbf{n}(p) \cdot \mathbf{N}(p)| \leq \mathbf{R}(p) \cdot \mathbf{N}(p)
$$

where $\mathbf{n}(p)$ is the unit normal to $M$ at $p, \mathbf{N}(p)$ is the outward unit normal to $\partial D$ at $p$ naturally included into $\mathbb{R}^{2} \times\{0\}$, and $\mathbf{R}(p)$ is the unit normal in the direction of $\mathcal{R}(p) \in \Upsilon$ emanating from $p$ also naturally included into $\mathbb{R}^{2} \times\{0\}$. Then $M$ has least area among all surfaces (graphs or not) having the same boundary as $M$.

Proof. Basic idea: Construct $\phi$ to be the calibration for $M$ that is dual to the tangent planes of $M$ and extend $\phi$ to the cylinder over $D$ so that $\phi$ is constant in the $z$ direction, as before. For comparison surfaces that go outside the cylinder, project onto the cylinder in the direction defined 
by the rays of $\Upsilon$. This projection stretches area (the bad news), but then integration of $\phi$ counts less than the projected area (the good news). The inequality in the hypothesis guarantees that the latter factor outweighs the former.

More formally, let $C$ be the cylinder $D \times \mathbb{R}$. Let $\Pi$ be the projection arising naturally from the choice of rays. That is, at points of $C, \Pi$ is the identity and at points away from $C, \Pi(x, y, z)=\left(x_{0}, y_{0}, z\right)$ where $(x, y) \in \mathcal{R}_{\alpha}$ and $\left(x_{0}, y_{0}\right)=\partial \mathcal{R}_{\alpha}$.

Now let $S$ be any surface (integral current) with the same boundary as $M$. Let $S_{1}=S\left\llcorner C\right.$, and $S_{2}=S-S_{1}$. Then

$$
\begin{aligned}
\mathbf{M}(S) & =\mathbf{M}\left(S_{1}\right)+\mathbf{M}\left(S_{2}\right) \\
\partial\left(\Pi_{\#} S\right) & =\Pi_{\#}(\partial S)=\partial S=\partial M \\
\mathbf{M}(M) & =\int_{M} \phi=\int_{\Pi_{\#} S} \phi=\int_{\Pi_{\#} S_{1}} \phi+\int_{\Pi_{\#} S_{2}} \phi \\
& =\int_{S_{1}} \phi+\int_{\Pi_{\#} S_{2}} \phi \leq \mathbf{M}\left(S_{1}\right)+\int_{\Pi_{\#} S_{2}} \phi .
\end{aligned}
$$

It remains to establish that

$$
\int_{\Pi_{\#} S_{2}} \phi \leq \mathbf{M}\left(\mathbf{S}_{2}\right)
$$

Since $\Pi_{\#} S_{2}$ lies on the boundary of $C$, and since $\phi$ is dual to the tangent planes of $M$, then $\phi$ applied to the tangent plane of $\Pi_{\#} S_{2}$ at a point $(p, z)=$ $\left(x_{0}, y_{0}, z\right)$ will equal $\pm \mathbf{n}(p) \cdot \mathbf{N}(p)$, where $\mathbf{n}(p)$ is a unit normal to $M$ at $(p, f(p))$ and (as before) $\mathbf{N}(p)$ is the outward unit normal to $\partial D$. Thus,

$$
\int_{\Pi_{\#} S_{2}} \phi \leq \int_{\Pi_{\#} S_{2}}|\mathbf{n}(p) \cdot \mathbf{N}(p)| \leq \int_{\Pi_{\#} S_{2}} \mathbf{R}(p) \cdot \mathbf{N}(p) .
$$

To compare the last integral with the mass of $S_{2}$, let $\mathcal{S}_{2}$ denote the support of the current $S_{2}, \mu(x)$ its multiplicity function and $J$ the Jacobian of $\Pi$. Define $g=1 / J$ when $J \neq 0$ and $g=0$ when $J=0$. From the definition of a nice complementary set of rays, $\mathbf{R}(p) \cdot \mathbf{N}(p)$ is defined and is bounded away from 0 for almost all $p \in \partial D$. Therefore $\Pi$ is Lipshcitz and it follows from the area-coarea formula that

$$
\begin{aligned}
\mathbf{M}\left(S_{2}\right)=\int_{S_{2}} \mu & \geq \int_{\mathcal{S}_{2}} g(x) J(x) \mu(x) d \mathcal{H}^{2} \\
& \geq \int_{\Pi\left\{\mathcal{S}_{2}\right\}} \sum_{x \in \Pi^{-1}\{p\} \cap \mathcal{S}_{2}} g(x) \mu(x) d \mathcal{H}^{2} .
\end{aligned}
$$

Note that the second inequality would be an equation if we used the (potentially smaller) Jacobian of the function $\Pi$ restricted to $\mathcal{S}_{2}$. 
Now if the rays $\mathcal{R}_{\alpha}$ near $p \in \partial D$ are parallel, then $J=1 /(\mathbf{R}(p) \cdot \mathbf{N}(p))$ on those rays, as seen in Figure 1. Otherwise the rays diverge (since they can't cross), so that $J \leq 1 /(\mathbf{R}(p) \cdot \mathbf{N}(p))$. Thus,

$$
\begin{aligned}
\int_{\Pi_{\#} S_{2}} \mathbf{R}(p) \cdot \mathbf{N}(p) & =\int_{\Pi\left\{\mathcal{S}_{2}\right\}} \mu \mathbf{R}(p) \cdot \mathbf{N}(p) d \mathcal{H}^{2} \\
& \leq \int_{\Pi\left\{\mathcal{S}_{2}\right\}} \sum_{x \in \Pi^{-1}\{p\} \cap \mathcal{S}_{2}} g(x) \mu(x) d \mathcal{H}^{2} .
\end{aligned}
$$

The last inequality may be strict for two reasons: If $\mathbf{R}(p) \cdot \mathbf{N}(p)<1 / J$, or if $\Pi_{\#}$ causes cancellation of sheets of $S_{2}$. Finally, there are only a countable number of points $p \in \partial D$ such that the Jacobian on $\Pi^{-1}(p)$ is zero, so even though $g=0<\mathbf{R}(p) \cdot \mathbf{N}(p)$ at these points, this does not affect the integral inequality.

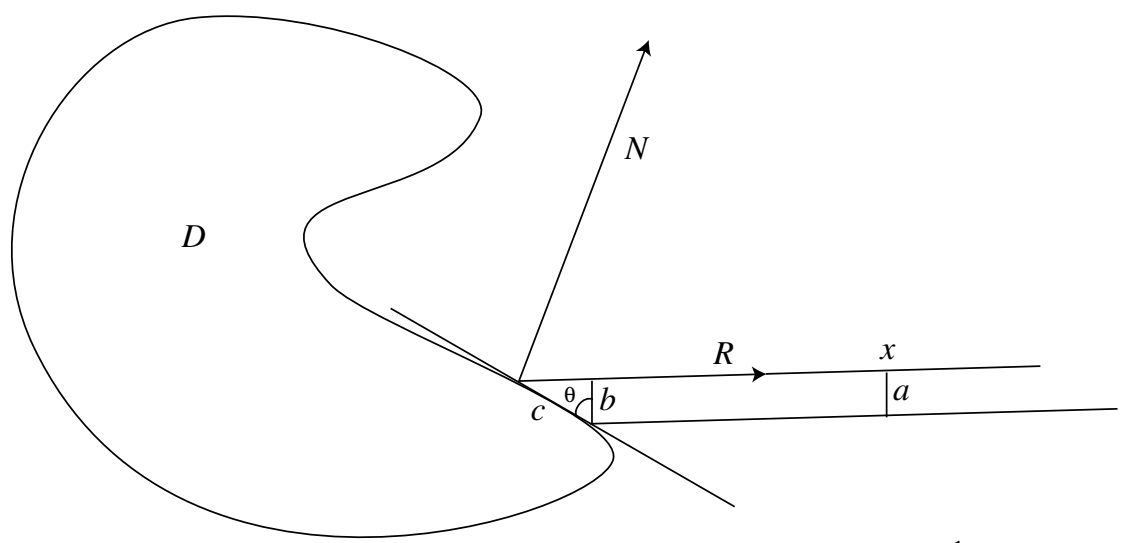

Figure 1. For parallel rays, $J_{1} \Pi(x)=\frac{c}{a} \leq \frac{c}{b}=\sec \theta=\frac{1}{\mathbf{N} \cdot \mathbf{R}}$. A similar picture in $\mathbb{R}^{3}$ shows that $J_{2} \Pi(x)=\frac{1}{\mathbf{N} \cdot \mathbf{R}}$.

In higher dimensions, it is not sufficient for the rays $\mathcal{R}_{\alpha}$ to be nonintersecting, because that does not guarantee that the distance between them is always increasing. (They might be skew, with points of closest approach somewhere away from $C$.) One must require that the rays be either parallel or diverging from each other, and then the theorem goes through as before.

We finish this section with some results that will allow us to more easily verify that a given set of rays satisfies the conditions of Theorem 3.2 in the applications of the next section.

Let $D$ be a closed domain. A set of non-crossing rays $\mu_{1}, \mu_{2} \ldots, \mu_{k}$ emanating from $\partial D$ so that $\mu_{i} \cap D=\partial \mu_{i}$ is said to partition the complement 
of $D$. The closures of the connected components of $\mathbb{R}^{2}-\left(D \cup \bigcup_{i=1}^{k} \mu_{i}\right)$ are called the sections of the partition.

Definition 3.3. Let $S$ be a section of the complement of a closed domain $D$ such that $C=\partial D \cap S$ is piecewise smooth. Suppose that $\Upsilon$ is a set of rays having the following properties:

(1) $\mathcal{R} \cap D=\partial \mathcal{R}$ for every $\mathcal{R} \in \Upsilon$.

(2) $\mathcal{R} \cap \mathcal{R}^{\prime} \subset C$ for every $\mathcal{R}, \mathcal{R}^{\prime} \in \Upsilon$.

(3) $S=\bigcup_{\mathcal{R} \in \Upsilon} \mathcal{R}$.

(4) There is a $\delta>0$ and a set $A \subset \partial D$ so that the one-dimensional Hausdorff measure $\mathcal{H}^{1}(A)$ equals 0 and for all $p \in \partial D-A$, the angle between $C$ and any ray $\mathcal{R} \in \Upsilon$ is defined and is at least $\delta$.

Then $\Upsilon$ is called a nice complementary set of rays for $D$ in $S$. If $\Upsilon$ satisfies (1)-(3), then $\Upsilon$ is called a complementary set of rays for $D$ in $S$

Given a ray $\mathcal{R}$ in $\mathbb{R}^{2}$, let $\vartheta(\mathcal{R})$ denote the direction of $\mathcal{R}$ with respect to the $x$-axis. The first proposition is an observation.

Propositon 3.4. Suppose that $D$ is a closed domain with connected boundary in $\mathbb{R}^{2}, \mu_{1}, \mu_{2} \ldots, \mu_{k}$ is a set of rays that partition the complement of $D$ so that $\vartheta\left(\mu_{1}\right) \leq \vartheta\left(\mu_{2}\right) \leq \cdots \leq \vartheta\left(\mu_{k}\right)$. Let $S_{i}$ denote the section of the partition bounded between $\mu_{i}$ and $\left.\mu_{(i+1} \bmod k\right)$. Suppose $\Upsilon_{i}$ is a (nice) complementary set of rays for $D$ in $S_{i}$. Then $\Upsilon=\bigcup \Upsilon_{i}$ is a (nice) complementary set of rays for $D$ in $\mathbb{R}^{2}$. Moreover, if $\mu_{1}, \mu_{2} \ldots, \mu_{k}$ partition the complement of $D$ into congruent sections then a given $\Upsilon_{i}$ extends to a (nice) complementary set of rays for $D$ in all of $\mathbb{R}^{2}$ via the congruence relation.

To determine if a particular set of rays in a section $S$ of the complement of a closed domain $D$ is a complementary set of rays for $D$ in $S$, we have the following definition and proposition:

Definition 3.5. Suppose that $D$ is a closed domain with connected boundary in $\mathbb{R}^{2}, \mu_{1}$ and $\mu_{2}$ are rays of a partition of the complement of $D$ that bounds a section $S$, and $\mathbf{R}_{0}$ is a unit vector that is in the direction of a ray contained in $S$. For each $p \in \partial D \cap S$, define $\mathcal{R}(p)$ to be a ray emanating from $p$ in the direction of $\mathbf{R}_{0}$. Let $W_{i}$ be the sector in $S$ cut out by the angle formed by $\mu_{i}$ and $\mathcal{R}\left(\partial \mu_{i}\right)$. Then the $\mathbf{R}_{0}$-set of rays for $D$ in $S$ is the union of the set $\{\mathcal{R}(p) \mid p \in \partial D \cap S\}$ together with the set of all rays emanating from $\partial \mu_{i}$ contained in $W_{i}$ for $i=0,1$.

Propositon 3.6. Let $D$ be a closed domain with connected boundary in $\mathbb{R}^{2}$. Suppose that $S$ is a section of a partition of the complement of $D$ bounded by $\mu_{1}$ and $\mu_{2}, C=\partial D \cap S$ is a smooth connected arc, $\mathbf{R}_{0}$ is a unit vector that is in the direction of a ray contained in $S$ and $\mathbf{N}(p) \cdot \mathbf{R}_{0}>0$ for all $p \in C-\partial C$. Then $\Upsilon$, the $\mathbf{R}_{0}$-set of rays for $C$ in $S$, is a complementary 
set of rays for $D$ in $S$. Moreover, if $\mathbf{N}(p) \cdot \mathbf{R}_{0}>0$ for all $p \in C$, then $\Upsilon$ is a nice complementary set of rays for $D$ in $S$.

Proof. The result will follow almost immediately from the next two lemmas.

Lemma 3.7. Suppose that $C$ is parameterized by $\mathbf{r}:\left[t_{1}, t_{2}\right] \rightarrow C$ and $l(p)$ is the line meeting $C$ at $p=\mathbf{r}\left(t_{0}\right)$ in the direction of $\mathbf{R}_{0}$. Define $\delta_{p}(q)$ to be the distance between $q$ and $l(p)$. Then $\delta_{p}\left(\mathbf{r}\left(t^{\prime}\right)\right) \geq \delta_{p}(\mathbf{r}(t))$ whenever $t^{\prime} \geq t \geq t_{0}$ or $t^{\prime} \leq t \leq t_{0}$.

Proof. Suppose not. Then there is a $t^{*} \in\left(t_{1}, t_{2}\right)$ so that $\delta_{p}$ has a local maximum at $\mathbf{r}\left(t^{*}\right)$. Then

$$
0=\delta_{p}^{\prime}\left(\mathbf{r}\left(t^{*}\right)\right)=\nabla \delta_{p}\left(\mathbf{r}\left(t^{*}\right)\right) \cdot \mathbf{r}^{\prime}\left(t^{*}\right) .
$$

Thus $\nabla \delta_{p}\left(\mathbf{r}\left(t^{*}\right)\right) \perp \mathbf{r}^{\prime}\left(t^{*}\right)$. In addition, $\nabla \delta_{p}\left(\mathbf{r}\left(t^{*}\right)\right) \perp l(p)$ since the direction of greatest increase of $\delta_{p}$ is perpendicular to $l(p)$. Therefore $l\left(\mathbf{r}\left(t^{*}\right)\right)$ is parallel to $\mathbf{r}^{\prime}\left(t^{*}\right)$ which contradicts that $\mathbf{N}\left(\mathbf{r}\left(t^{*}\right)\right) \cdot \mathbf{R}_{0}>0$.

Lemma 3.8. The map $p \rightarrow l(p)$ is a 1-1 correspondence between the points of $C$ and the parallel lines bounded between $l\left(a_{1}\right)$ and $l\left(a_{2}\right)$.

Proof. It follows from Lemma 3.7 that no two distinct points of $C$ lie on the same line bounded between $l\left(a_{1}\right)$ and $l\left(a_{2}\right)$. Thus $C$ is contained in the region bounded between $l\left(a_{1}\right)$ and $l\left(a_{2}\right)$. By the connectedness of $C$, each of the parallel lines bounded between $l\left(a_{1}\right)$ and $l\left(a_{2}\right)$ meets $C$. Thus the map that assigns each point $p$ of $C$ to the line $l(p)$ containing $p$ and bounded between $l\left(a_{1}\right)$ and $l\left(a_{2}\right)$ is a 1-1 correspondence.

For each $p \in C$, the ray $\mathcal{R}(p)$ is the ray with endpoint $p$ that is contained in $l(p)$ and emanating from away from $D$. Hence $\mathcal{R}(p) \cap D=\partial \mathcal{R}(p)$. Since $D$ is a domain with connected boundary then $W_{i}$ can meet $D$ only at $a_{i}$. Thus for $\mathcal{R}$ emanating from $a_{i}$ and contained in $W_{i}$, it is again the case that $\mathcal{R} \cap D=\partial \mathcal{R}$. Therefore Condition 1 is satisfied.

Let $T$ be that part of the region bounded by $l\left(a_{1}\right), l\left(a_{2}\right)$ and $C$ which misses the interior of $D$. Then $S=W_{1} \cup W_{2} \cup T$. It should be clear from Lemma 3.8 that $S=\bigcup_{\mathcal{R} \in \Upsilon} \mathcal{R}$ and that $\mathcal{R} \cap \mathcal{R}^{\prime} \subset C$ for every $\mathcal{R}, \mathcal{R}^{\prime} \in \Upsilon$. Therefore, Conditions 2 and 3 are satisfied.

If $\mathbf{N}(p) \cdot \mathbf{R}_{0}>0$ for all $p \in C$ then Condition 4 follows from the compactness of $C$.

In the applications of the next section we will identify partitions that divide a given domain $D$ into congruent pieces. Then for a specified section

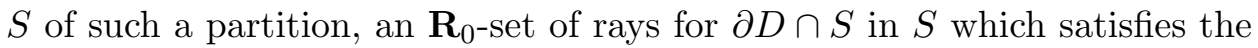
conditions of Proposition 3.6 is determined. Applying Proposition 3.4, this set of rays is extended to a nice complementary set of rays for $D$ in $\mathbb{R}^{2}$. 


\section{Applications.}

Theorem 3.2 allows us to prove that certain minimal graphs over nonconvex domains are area-minimizing. For our first application we will show that a particular portion of Enneper's surface minimizes area. Recently, White proved that half of Enneper's surface is area-minimizing [15], and previously, we mentioned that Enneper's surface is area-minimizing over $\pi_{r_{0}}$ for $r_{0}=\sqrt{2^{2 / 3}-1} \approx 0.766$. Using Theorem 3.2 , we are able to establish that Enneper's surface is area-minimizing over $\pi_{r_{0}}$ for $r_{0}=\frac{1}{3} \sqrt{3+2 \sqrt{3}} \approx 0.847$ (see Figures 2 and 3).

Theorem 4.1. Enneper's surface minimizes area among all surfaces spanning the curve $C=\left\{X(u, v) \mid u^{2}+v^{2}=r_{0}^{2}\right\}$ where $X(u, v)$ is given by Equation (1) and $r_{0}=\frac{1}{3} \sqrt{3+2 \sqrt{3}}$.

Proof. Using polar coordinates so that $(u, v)=(r \cos \theta, r \sin \theta)$, the first two coordinates for Enneper's surface are given by

$$
\begin{aligned}
& x=r \cos \theta+r^{3} \cos \theta-\frac{4}{3} r^{3} \cos ^{3} \theta \\
& y=r \sin \theta+r^{3} \sin \theta-\frac{4}{3} r^{3} \sin ^{3} \theta .
\end{aligned}
$$

Let $\mathbf{n}$ denote the surface normal to Enneper's surface and $\mathbf{N}$ the outward unit normal to $\partial \pi_{r}$ for a fixed $r$. Then

$$
\begin{aligned}
& \mathbf{N}=\frac{\left(\cos \theta\left(1-3 r^{2}+4 r^{2} \cos ^{2} \theta\right), \sin \theta\left(1-3 r^{2}+4 r^{2} \sin ^{2} \theta\right), 0\right)}{\sqrt{\left(r^{2}+1\right)^{2}-16 r^{2} \cos ^{2} \theta \sin ^{2} \theta}}, \text { and } \\
& \mathbf{n}=\frac{1}{1+r^{2}}\left(2 r \cos \theta,-2 r \sin \theta, r^{2}-1\right) .
\end{aligned}
$$

Let $\theta_{i}=\frac{(2 i-1)}{4} \pi$ for $i=0,1,2,3$. It is a straightforward calculation to show that $X(r \cos \theta, r \sin \theta)$ meets the ray emanating from the origin in the $\theta_{i}$ direction precisely when $\theta=\theta_{i}$ and that the distance from $X\left(r \cos \theta_{i}, r \sin \theta_{i}\right)$ to the origin increases in $r$. For a fixed $r$, let $\mu_{i}$ be the ray in the direction of $\theta_{i}$ emanating from $X\left(r \cos \theta_{i}, r \sin \theta_{i}\right)$ for $i=0,1,2,3$. Then $\left\{\mu_{i}\right\}$ partitions the complement of $\pi_{r}$ into congruent pieces. Let $S_{1}$ be the section of $\mathbb{R}^{2}$ bounded between $\mu_{0}$ and $\mu_{1}$. Note that $C_{1}=S \cap \partial \pi_{r}=$ $\{X(r \cos \theta, r \sin \theta) \mid-\pi / 4 \leq \theta \leq \pi / 4\}$ is a smooth curve.

Let $\mathbf{R}_{0}=(1,0,0)$ and let $\Upsilon_{1}$ be the $\mathbf{R}_{0}$-set of rays for $\pi_{r}$ in $S_{1}$. Observe that for $p \in \partial C_{1}$,

$$
\mathbf{N}(p) \cdot \mathbf{R}_{0}=\frac{\cos \theta\left(1-3 r^{2}+4 r^{2} \cos ^{2} \theta\right)}{\sqrt{\left(r^{2}+1\right)^{2}-16 r^{2} \cos ^{2} \theta \sin ^{2} \theta}} \geq \frac{1-r^{2}}{\sqrt{2}\left(1+r^{2}\right)} .
$$


Hence, by Proposition 3.6, $\Upsilon_{1}$ is a nice complementary set of rays for $\pi_{r}$ in $S_{1}$ when $r<1$. By Proposition 3.4, $\Upsilon_{1}$ extends to $\Upsilon$, a nice complementary set of rays for $\pi_{r}$ in $\mathbb{R}^{2}$.

In order to apply Theorem 3.2 to $\Upsilon$, we want to find the largest value of $r>0$ for which

$$
|\mathbf{n} \cdot \mathbf{N}| \leq \mathbf{N} \cdot \mathbf{R}
$$

almost everywhere on $\partial \pi_{r}$. Note that

$$
\mathbf{n} \cdot \mathbf{N}=\frac{2 r\left(2 \cos ^{2} \theta-1\right)}{\sqrt{\left(r^{2}+1\right)^{2}-16 r^{2} \cos ^{2} \theta \sin ^{2} \theta}} \geq 0 .
$$

Thus, we want to find the largest region $\pi_{r}$ so that $\mathbf{N} \cdot\left(\mathbf{R}_{\mathbf{0}}-\mathbf{n}\right) \geq 0$ holds. In particular, we want $r$ to satisfy

$$
\cos \theta\left(1-3 r^{2}+4 r^{2} \cos ^{2} \theta\right)-2 r\left(\cos ^{2} \theta-\sin ^{2} \theta\right) \geq 0
$$

for $\theta \in[-\pi / 4, \pi / 4]$. The value of $\theta \in[-\pi / 4, \pi / 4]$ that minimizes the lefthand side of (3) satisfies

$$
\cos \theta=\frac{2+\sqrt{1+9 r^{2}}}{6 r} .
$$

Substituting this value into (3), and solving for $r$ we get that

$$
r_{0}=\frac{1}{3} \sqrt{3+2 \sqrt{3}} \approx 0.84748 .
$$

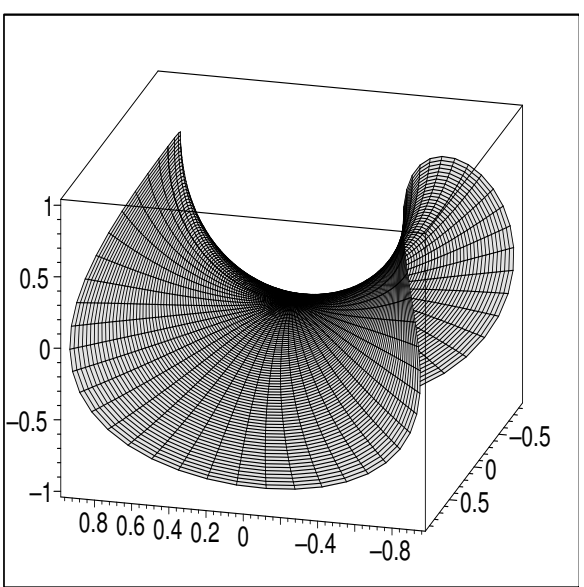

Figure 2. Enneper's surface with $r=1$.

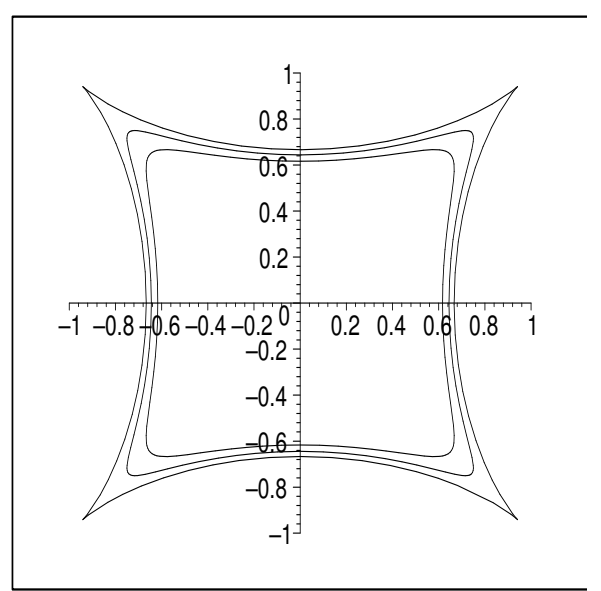

Figure 3. Projection of Enneper's surface with $r_{1}=0.767, r_{2}=0.847$, and $r_{3}=1$. 
Next, we apply Theorem 3.2 to determine portions of the Scherk singly periodic surface that are the minimal graphs over nonconvex domains which are area-minimizing. The Scherk singly periodic surface arises in minimal surface theory as the conjugate surface of the Scherk doubly periodic surface. Recall the following definitions:

Definition 4.2. If a minimal surface $X(u, v)=(x, y, z)$ is defined on a simply connected domain $\Omega \in \mathbb{C}$, then we define the conjugate surface or adjoint surface, $X^{*}(u, v)=\left(x^{*}, y^{*}, z^{*}\right)$ to $X(u, v)$ on $\Omega$ as the solution of the Cauchy-Riemann equations

$$
X_{u}=X_{v}^{*}, \quad X_{v}=-X_{u}^{*}
$$

in $\Omega$.

Example 4.3. The doubly periodic Scherk surface (see Figure 4) is given by

$$
X(u, v)=\left(\frac{1}{2} \arg \left(\frac{z-i}{z+i}\right), \frac{1}{2} \arg \left(\frac{1+z}{1-z}\right), \frac{1}{2} \log \left|\frac{1+z^{2}}{1-z^{2}}\right|\right)
$$

where $z=u+i v$. Its conjugate surface is the singly periodic Scherk surface (see Figure 5) given by

$$
X^{*}(u, v)=\left(\frac{1}{2} \log \left|\frac{z+i}{z-i}\right|, \frac{1}{2} \log \left|\frac{1-z}{1+z}\right|, \frac{1}{2} \arg \left(\frac{1+z^{2}}{1-z^{2}}\right)\right) .
$$

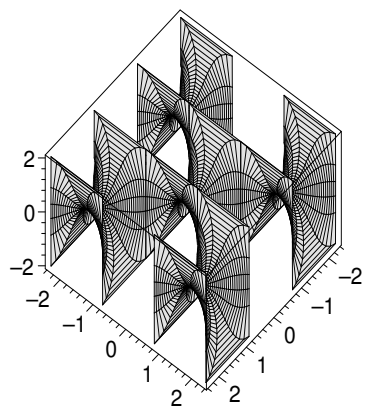

Figure 4. The doubly periodic Scherk surface with $r=0.999$.

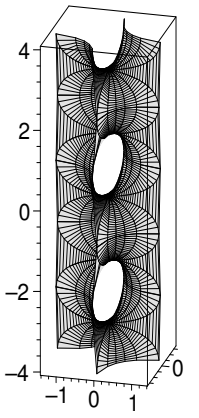

Figure 5. The singly periodic Scherk surface with $r=0.999$.

Now let $\pi_{r}^{0}$ denote the projection of that portion of the doubly periodic Scherk surface defined on $U_{r}$ to the $x, y$-plane. Likewise, let $\pi_{r}^{*}$ denote the projection of that portion of the singly periodic Scherk surface defined on $U_{r}$ to the $x, y$-plane. Since the doubly periodic Scherk surface is a graph over $\pi_{1}^{0}$ which is convex (see Figure 6), it is area-minimizing by Theorem 2.2. By a result by Krust [see [7] or [2]], the singly periodic Scherk surface is a minimal 
graph over $\pi_{1}^{*}$. The region $\pi_{1}^{*}$ is nonconvex (see Figure 7 ) and it has been unknown whether or not it is area-minimizing over $\pi_{r}^{*}$ for $r>1 / \sqrt{2} \approx 0.707$; i.e., for values $r$ such that the convex hull of $\pi_{r}^{*}$ is contained in $\pi_{1}^{*}$. However, $\pi_{r}^{*}$ has a nice complementary set of rays for $r<1$. Thus we can apply Theorem 3.2 to derive the following result:

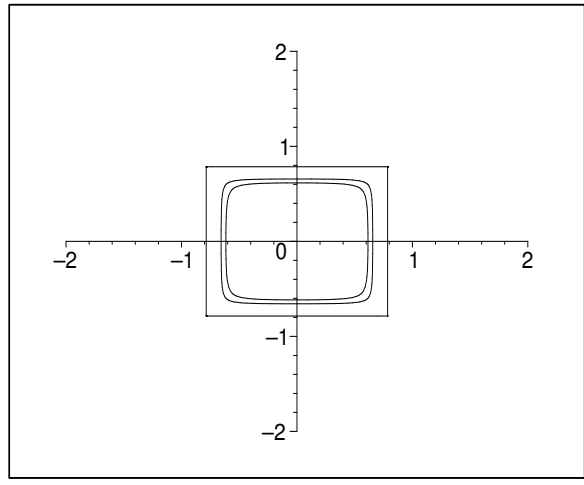

Figure 6. Projection of one component of the doubly periodic Scherk surface with $r_{1}=\frac{1}{\sqrt{2}}$, $r_{2}=0.770778$ and $r_{3}=0.999$.

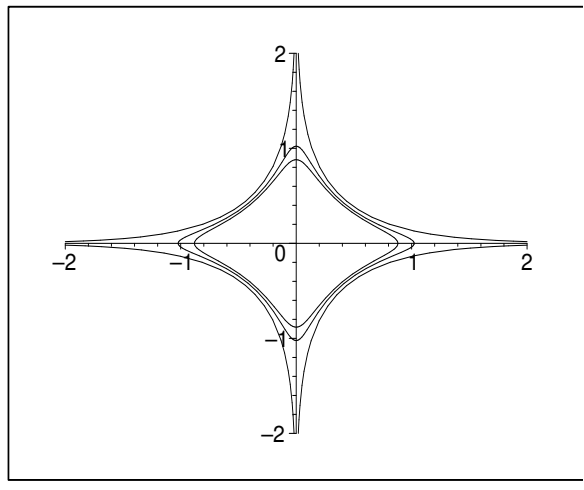

Figure 7. Projection of the singly periodic Scherk surface with $r_{1}=\frac{1}{\sqrt{2}}, r_{2}=0.770778$
and $r_{3}=0.999$

Theorem 4.4. The singly periodic Scherk surface minimizes area among all surfaces spanning the curve $C=\left\{X(u, v) \mid u^{2}+v^{2}=r_{0}^{2}\right\}$ where $X(u, v)$ is given by Equation (4) and

$$
\begin{aligned}
r_{0} & =\frac{1}{2} \sqrt{-10-2 \sqrt{17}+8 \sqrt{4+\sqrt{17}}}-2 \sqrt{102+26 \sqrt{17}-40 \sqrt{4+\sqrt{17}}-8 \sqrt{17} \sqrt{4+\sqrt{17}}} \\
& \approx 0.770778 .
\end{aligned}
$$

Proof. Parametrizing the $u, v$-plane in polar coordinates so that $(u, v)=$ $(r \cos \theta, r \sin \theta)$, we have

$$
\begin{aligned}
& x=\frac{1}{4} \log \left(\frac{r^{2}+1+2 r \sin \theta}{r^{2}+1-2 r \sin \theta}\right), \\
& y=\frac{1}{4} \log \left(\frac{r^{2}+1-2 r \cos \theta}{r^{2}+1+2 r \cos \theta}\right) .
\end{aligned}
$$

Let $\mathbf{n}$ denote the surface normal to the singly periodic Scherk surface and $\mathbf{N}$ the outward unit normal to $\partial \pi_{r}^{*}$ for a fixed $r$. Then

$$
\mathbf{N}=\frac{\left(\sin \theta\left[\left(1-r^{2}\right)^{2}+(2 r \cos \theta)^{2}\right],-\cos \theta\left[\left(1-r^{2}\right)^{2}+(2 r \sin \theta)^{2}\right], 0\right)}{\sqrt{\left(1-r^{2}\right)^{4}+16 r^{2} \cos ^{2} \theta \sin ^{2} \theta\left(1-r^{2}+r^{4}\right)}},
$$


and

$$
\mathbf{n}=\frac{-1}{1+r^{2}}\left(2 r \cos \theta,-2 r \sin \theta, r^{2}-1\right) .
$$

Let $\theta_{i}=\frac{i \pi}{2}$ for $i=0,1,2,3$. It is a straightforward calculation to show that $X(r \cos \theta, r \sin \theta)$ meets the ray emanating from the origin in the $\theta_{i}$ direction precisely when $\theta=\theta_{i}+\frac{\pi}{2}$ and that the distance from $X\left(r \cos \theta_{i}, r \sin \theta_{i}\right)$ to the origin increases in $r$. For a fixed $r$, let $\mu_{i}$ be the ray in the direction of $\theta_{i}$ emanating from $X\left(r \cos \left(\theta_{i}+\frac{\pi}{2}\right), r \sin \left(\theta_{i}+\frac{\pi}{2}\right)\right)$ for $i=0,1,2,3$. Then $\left\{\mu_{i}\right\}$ partitions the complement of $\pi_{r}$ into congruent pieces. Let $S_{1}$ be the section of $\mathbb{R}^{2}$ bounded between $\mu_{0}$ and $\mu_{1}$. Note that $C_{1}=S \cap \partial \pi_{r}=\left\{X(r \cos \theta, r \sin \theta) \mid \frac{\pi}{2} \leq \theta \leq \pi\right\}$ is a smooth curve.

Let $\mathbf{R}_{0}=(1 / \sqrt{2}, 1 / \sqrt{2}, 0)$ and let $\Upsilon_{1}$ be the $\mathbf{R}_{0}$ set of rays for $\partial \pi_{r} \cap S_{1}$ in $S_{1}$. Observe that for $p \in \partial \pi_{r} \cap S_{1}$,

$$
\begin{aligned}
\mathbf{N}(p) \cdot \mathbf{R}_{0} & =\frac{\sin \theta\left[\left(1-r^{2}\right)^{2}+(2 r \cos \theta)^{2}\right]-\cos \theta\left[\left(1-r^{2}\right)^{2}+(2 r \sin \theta)^{2}\right]}{\sqrt{2} \sqrt{\left(1-r^{2}\right)^{4}+16 r^{2} \cos ^{2} \theta \sin ^{2} \theta\left(1-r^{2}+r^{4}\right)}} \\
& \geq \frac{1}{\sqrt{2}} .
\end{aligned}
$$

Hence by Proposition 3.6, $\Upsilon_{1}$ is a nice complementary set of rays for $\pi_{r}^{*} \cap S_{1}$ in $S_{1}$. By Proposition 3.4, $\Upsilon_{1}$ extends to $\Upsilon$, a nice complementary set of rays for $\pi_{r}$ in $\mathbb{R}^{2}$.

We want to find the largest value of $r$ so that $|\mathbf{n} \cdot \mathbf{N}| \leq \mathbf{N} \cdot \mathbf{R}$. Note that for $\theta \in[\pi / 2, \pi]$

$$
\mathbf{n} \cdot \mathbf{N}=\frac{-4 r \cos \theta \sin \theta\left(1+r^{4}\right)}{\left(1+r^{2}\right) \sqrt{\left(1-r^{2}\right)^{4}+16 r^{2} \cos ^{2} \theta \sin ^{2} \theta\left(1-r^{2}+r^{4}\right)}} \geq 0 .
$$

Thus we want to find the largest region $\pi_{r}$ so that $\mathbf{N} \cdot(\mathbf{R}-\mathbf{n}) \geq 0$. In particular, we want to satisfy

$$
\begin{aligned}
& \left(1+r^{2}\right)(\sin \theta-\cos \theta)\left[\left(1-r^{2}\right)^{2}-4 r^{2} \cos \theta \sin \theta\right] \\
& +4 \sqrt{2} r \cos \theta \sin \theta\left(1+r^{4}\right) \geq 0
\end{aligned}
$$

for $\theta \in[\pi / 2, \pi]$. Differentiating and using the identity $\cos \theta \sin \theta=\frac{1}{2}[1-$ $\left.(\cos \theta-\sin \theta)^{2}\right]$ we find that the value of $\theta$ that minimizes the left-hand side of this inequality satisfies the equation

$$
\cos \theta-\sin \theta=\frac{-2 \sqrt{2}\left(1+r^{4}\right) \pm \sqrt{2 r^{8}+12 r^{6}+52 r^{4}+12 r^{2}+2}}{6 r\left(1+r^{2}\right)} .
$$


Substituting this value into (5) yields

$$
\begin{aligned}
\frac{\sqrt{2}}{54 r\left(1+r^{2}\right)^{2}}\left[( 1 + r ^ { 4 } ) \left(r^{8}+36 r^{6}+\right.\right. & \left.38 r^{4}+36 r^{2}+1\right) \\
& \left. \pm\left(r^{8}+6 r^{6}+26 r^{4}+6 r^{2}+1\right)^{\frac{3}{2}}\right] \geq 0 .
\end{aligned}
$$

Hence, we need the expression inside of the square brackets to be greater than 0 . This leads to finding the smallest positive root of

$$
54 r^{2}\left(r^{2}+1\right)^{2}\left[r^{16}+20 r^{14}-8 r^{12}+28 r^{10}-146 r^{8}+28 r^{6}-8 r^{4}+20 r^{2}+1\right] .
$$

Factoring $r^{8}$ out of the expression in the square brackets and substituting in

$$
t=r^{2}+\frac{1}{r^{2}}
$$

gives the new expression in the square brackets as

$$
t^{4}+20 t^{3}-12 t^{2}-32 t-128,
$$

whose roots are $-5-\sqrt{17} \pm 4 \sqrt{4+\sqrt{17}}$ and $-5+\sqrt{17} \pm 4 \sqrt{4-\sqrt{17}}$. The smallest positive root of

$$
r^{16}+20 r^{14}-8 r^{12}+28 r^{10}-146 r^{8}+28 r^{6}-8 r^{4}+20 r^{2}+1
$$

is therefore

$r_{0}=\frac{1}{2} \sqrt{-10-2 \sqrt{17}+8 \sqrt{4+\sqrt{17}}-2 \sqrt{102+26 \sqrt{17}-40 \sqrt{4+\sqrt{17}}-8 \sqrt{17} \sqrt{4+\sqrt{17}}}}$

In general, a conjugate surface is a minimal surface. Thus, we can construct a one-parameter family of minimal surfaces.

Definition 4.5. For $\alpha \in \mathbb{R}$, the surfaces $Z(u, v, \alpha)$ are called associated minimal surfaces to the surface $X(u, v)$, where

$$
Z(u, v, \alpha):=X(u, v) \cos \alpha+X^{*}(u, v) \sin \alpha .
$$

Associated surfaces share several nice properties. In the references above, Krust showed that if an embedded minimal surface can be written as a graph over a convex domain, then all associated minimal surfaces are graphs. Recently, Dorff [3] proved that these associated surfaces are graphs over close-to-convex domains.

Example 4.6. The associated surface of the doubly periodic Scherk surface for $\alpha=\pi / 4$ along with its projection onto a close-to-convex domain are shown in Figures 8 and 9. 


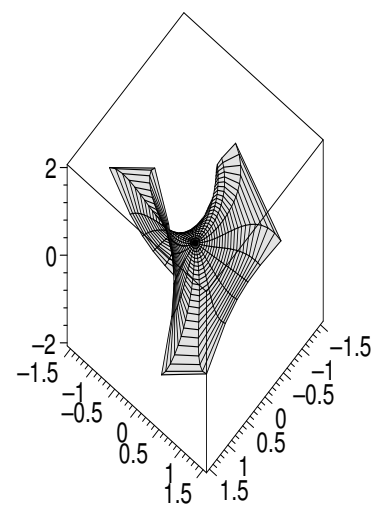

Figure 8. Associated Scherk surface for $\alpha=\pi / 4$ with $r=0.999$.

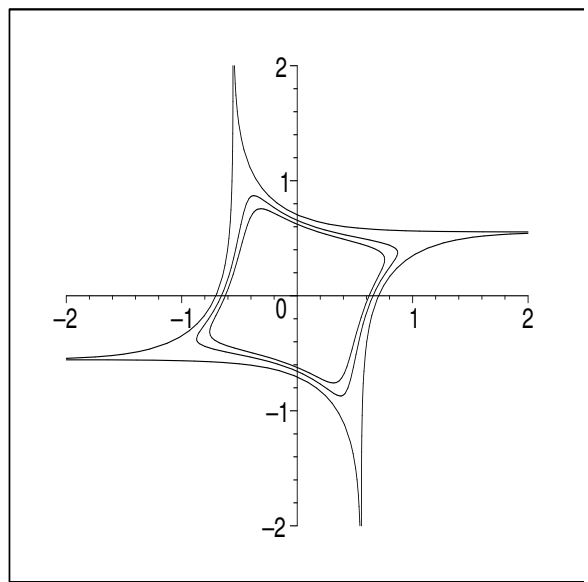

Figure 9. Projection of associated Scherk surface for $\alpha=\pi / 4$ with $r_{1}=0.7, r_{2}=0.77$, and $r_{3}=0.999$.

Using Theorem 3.2 and a computer assisted approach for proving inequalities, we now determine a whole class of subsurfaces of the Scherk associated surfaces that are graphs over nonconvex domains and are area-minimizing.

Theorem 4.7. For $0 \leq \alpha \leq \frac{\pi}{2}$, the associated Scherk surface minimizes area among all surfaces spanning the curve $C_{\alpha}=\left\{Z(u, v, \alpha) \mid u^{2}+v^{2}=r_{0}^{2}\right\}$ where $Z(u, v, \alpha)$ is given by Equation (6) and $r_{0}=0.77043$

Proof. Let the $u, v$-plane be parameterized in polar coordinates so that $(u, v)=(r \cos \theta, r \sin \theta)$. Note that the normal $\mathbf{N}_{\alpha}$ to $C_{\alpha}$ in the plane $z=0$ is given by

$$
\mathbf{N}_{\alpha}=\frac{\widetilde{\mathbf{N}}_{\alpha}}{\left\|\widetilde{\mathbf{N}}_{\alpha}\right\|}
$$

where

$$
\widetilde{\mathbf{N}}_{\alpha}=\cos \alpha \mathbf{N}_{0}+\sin \alpha \mathbf{N}_{\frac{\pi}{2}} \text {. }
$$

It follows that

$$
\begin{aligned}
\widetilde{\mathbf{N}}_{\alpha}= & \cos \alpha\left(\frac{r\left(1-r^{2}\right) \cos \theta}{\left[\left(1-r^{2}\right)^{2}+(2 r \sin \theta)^{2}\right]}, \frac{r\left(1-r^{2}\right) \sin \theta}{\left[\left(1-r^{2}\right)^{2}+(2 r \cos \theta)^{2}\right]}, 0\right) \\
& +\sin \alpha\left(\frac{r\left(r^{2}+1\right) \sin \theta}{\left[\left(1-r^{2}\right)^{2}+(2 r \sin \theta)^{2}\right]}, \frac{-r\left(r^{2}+1\right) \cos \theta}{\left[\left(1-r^{2}\right)^{2}+(2 r \cos \theta)^{2}\right]}, 0\right), \text { and } \\
\mathbf{n}= & \frac{-1}{1+r^{2}}\left(2 r \cos \theta,-2 r \sin \theta, r^{2}-1\right) .
\end{aligned}
$$

To construct a nice complementary set of rays for $\pi_{r}^{\alpha}$ that satisfies the conditions of Theorem 3.2, we first identify a simple partition of the complement 
of $\pi_{r}^{\alpha}$ from which we construct a complementary set of rays for $\pi_{r}^{\alpha}$. A finite subset of these rays then gives a different partition of the complement of $\pi_{r}^{\alpha}$. Finally, we utilize the new partition to define a nice complementary set of rays for $\pi_{r}^{\alpha}$.

To obtain our first partition, note that the maximum $x$-coordinate for points of $\pi_{r}^{\alpha}$ occurs when the $y$ component of $\mathbf{N}_{\alpha}$ is 0 . This occurs when

$$
\tan \theta=\frac{1+r^{2}}{1-r^{2}} \tan \alpha \text {. }
$$

Let

$$
\theta_{i}^{*}=\tan ^{-1}\left[\frac{1+r^{2}}{1-r^{2}} \tan \alpha\right]+\frac{\pi i}{2} \text { for } i=0,1,2,3 .
$$

Let $\phi_{i}=\frac{\pi i}{2}$ for $i=0,1,2,3$. Let $\hat{Z}$ denote the orthogonal projection of the map $Z$ to the $x y$-plane. Let $\mu_{i}^{*}$ be the ray in the $\phi_{i}$ direction emanating from $\hat{Z}\left(r \cos \theta_{i}^{*}, r \sin \theta_{i}^{*}, \alpha\right)$. Then clearly $\left\{\mu_{i}^{*}\right\}$ partitions the complement of $\pi_{r}^{\alpha}$ into congruent sections. Let $S_{0}^{*}$ be the section bounded between $\mu_{0}^{*}$ and $\mu_{1}^{*}$. Note that

$$
C_{0}^{*}=S_{0} \cap \partial \pi_{r}^{\alpha}=\left\{\hat{Z}(r \cos \theta, r \sin \theta, \alpha) \mid \theta_{0}^{*}<\theta<\theta_{1}^{*}\right\}
$$

is a smooth curve. Let $\mathbf{R}^{*}=(1,0,0)$. Then

$$
\mathbf{N}_{\alpha} \cdot \mathbf{R}^{*}=\frac{r\left(1-r^{2}\right) \cos \alpha \cos \theta+r\left(r^{2}+1\right) \sin \alpha \sin \theta}{\left\|\widetilde{\mathbf{N}}_{\alpha}\right\|\left[\left(1-r^{2}\right)^{2}+(2 r \sin \theta)^{2}\right]} .
$$

For $\theta_{0}^{*} \leq \theta \leq \frac{\pi}{2}$, it is clear that $\mathbf{N}_{\alpha} \cdot \mathbf{R}^{*}>0$. For $\theta_{1}^{*} \leq \theta<\frac{\pi}{2}$ we have

$$
-\cos \theta<\frac{1+r^{2}}{1-r^{2}} \tan \alpha \sin \theta .
$$

It follows that $\mathbf{N}_{\alpha} \cdot \mathbf{R}^{*}>0$ for $\frac{\pi}{2} \leq \theta \leq \theta_{1}^{*}$. (In fact $\mathbf{N}_{\alpha} \cdot \mathbf{R}^{*}=0$ at $\theta=\theta_{1}^{*}$.) Hence the $\mathbf{R}^{*}$-set of rays for $\pi_{r}^{\alpha}$ in $S_{0}^{*}$ extends to a complementary set of rays $\Upsilon^{*}$ for $\pi_{r}^{\alpha}$. Note that any finite set of rays in $\Upsilon^{*}$ forms a partition of the complement of $\pi_{r}^{\alpha}$.

Now let

$$
\omega(\alpha)=\cos ^{-1}\left[-\frac{19}{64}+\frac{7}{3} \cos \frac{\alpha}{2}-\frac{4}{3} \cos ^{2} \frac{a}{2}-\frac{17}{10} \sin \frac{a}{2}+\sin ^{2} \frac{a}{2}\right] .
$$

It can be shown by the computer assisted method of the next section that for $\alpha \in\left[0, \frac{\pi}{2}\right]$,

$$
\theta_{0}^{*} \leq \omega(\alpha) \leq \theta_{1}^{*} .
$$

Let $\omega_{i}=\omega(\alpha)+\frac{\pi i}{2}$ for $i=0,1,2,3$. Let $\mu_{i}$ be the ray emanating from $\hat{Z}\left(r \cos \omega_{i}, r \sin \omega_{i}, \alpha\right)$ in the $\phi_{i}$ direction. Then $\left\{\mu_{i}\right\} \subset \Upsilon^{*}$ and hence $\left\{\mu_{i}\right\}$ 
partitions the complement of $\pi_{r}^{\alpha}$. Let $S_{0}$ be the section of the complement bounded between $\mu_{0}$ and $\mu_{1}$. Note that

$$
C_{0}=S_{0} \cap \partial \pi_{r}^{\alpha}=\left\{\hat{Z}(r \cos \theta, r \sin \theta, \alpha) \mid \omega_{0}<\theta<\omega_{1}\right\}
$$

is a smooth curve. Let $\mathbf{R}_{\alpha}=\left(\sin \frac{\alpha}{2}, \cos \frac{\alpha}{2}, 0\right)$. Again, by using the computer assisted approach of the next section, it can be shown that for $\alpha \in\left[0, \frac{\pi}{2}\right], \theta \in\left[\omega_{0}, \omega_{1}\right]$ and $r_{0}=0.77043$ that

$$
\mathbf{N}_{\alpha} \cdot \mathbf{R}_{\alpha}-\left|\mathbf{n} \cdot \mathbf{N}_{\alpha}\right|>0 .
$$

Hence the $\mathbf{R}_{\alpha}$-set of rays for $\pi_{r}^{\alpha}$ in $S_{0}$ extends to a nice complementary set of rays for $\pi_{r_{0}}^{\alpha}$. Moreover, the hypothesis of Theorem 3.2 is satisfied for $r_{0}=0.77043$ and all $\alpha \in\left[0, \frac{\pi}{2}\right]$. Therefore, for $0 \leq \alpha \leq \frac{\pi}{2}$, the associated Scherk surface minimizes area among all surfaces spanning the curve $C_{\alpha}=\left\{Z(u, v, \alpha) \mid u^{2}+v^{2}=r_{0}^{2}\right\}$ where $r_{0}=0.77043$.

\section{A computer assisted approach to proving inequalities.}

We begin with a simple observation about polynomials: If a polynomial is dominated by its constant coefficient, then for small $x$ the value is always positive or always negative. More specifically:

Lemma 5.1. Let $P(x)=a_{0}+a_{1} x+\cdots+a_{n} x^{n}$. Suppose that

$$
\left|a_{0}\right|>\left|a_{1}\right|+\left|a_{2}\right|+\cdots+\left|a_{n}\right| .
$$

Then $P(x)$ has the same sign as $a_{0}$ for all $x \in[-1,1]$.

Proof. Let $x \in[-1,1]$. Then

$$
\begin{aligned}
a_{0} P(x)-a_{0}^{2} & =a_{0}\left(a_{1} x+\cdots+a_{n} x^{n}\right) \\
& \geq-\left|a_{0}\right|\left|a_{1} x+\cdots+a_{n} x^{n}\right| \\
& \geq-\left|a_{0}\right|\left(\left|a_{1}\right||x|+\cdots+\left|a_{n}\right|\left|x^{n}\right|\right) \\
& \geq-\left|a_{0}\right|\left(\left|a_{1}\right|+\cdots+\left|a_{n}\right|\right) .
\end{aligned}
$$

Thus

$$
\frac{a_{0}}{\left|a_{0}\right|} P(x) \geq\left|a_{0}\right|-\left(\left|a_{1}\right|+\cdots+\left|a_{n}\right|\right)>0 .
$$

This gives a method for proving a polynomial inequality for $x \in[-1,1]$. We now extend Lemma 5.1 in order to prove polynomial inequalities for more general intervals $x \in\left[x_{0}-t, x_{0}+t\right]$. 
Lemma 5.2. Let $P(x)=a_{0}+a_{1} x+\cdots+a_{n} x^{n}$, and for some fixed $t>0$ and $x_{0} \in \mathbf{R}$ let $Q(x)=P\left(x t+x_{0}\right)$. Suppose that when expanded out, $Q(x)=b_{0}+b_{1} x+\cdots+b_{n} x^{n}$ and that $\left|b_{0}\right|$ dominates the sum of absolute values of the other coefficients $b_{i}$ as in Lemma 5.1. Then $P(x)$ has the same sign as $b_{0}$ for all $x \in\left[x_{0}-t, x_{0}+t\right]$.

Proof. By Lemma 5.1, $\frac{b_{0}}{\left|b_{0}\right|} Q(x)>0$ for all $x \in[-1,1]$. Set $s=x t+x_{0}$, so that when $x \in[-1,1], s \in\left[x_{0}-t, x_{0}+t\right]$. Since $Q(x)=P\left(x t+x_{0}\right)$, we get that $\frac{b_{0}}{\left|b_{0}\right|} Q(x)=\frac{b_{0}}{\left|b_{0}\right|} P(s)>0$ for all $s \in\left[x_{0}-t, x_{0}+t\right]$, as desired.

Lemma 5.2 gives a method by which a computer can prove a polynomial inequality on a compact interval $I$. Divide $I$ into small pieces. Notice that the smaller $t$ is, the more likely $b_{0}$ is to dominate the other coefficients of $Q(x)=P\left(x t+x_{0}\right)$, since the other coefficients all get multiplied by a positive power of $t$. If $P$ is strictly positive on all of $I$, then this method will work for a sufficiently fine subdivision.

Next we deal with polynomials of more than one variable.

Lemma 5.3. Let $\mathbf{x}=\left(x_{1}, \ldots, x_{k}\right)$, and let $P(\mathbf{x})$ be a polynomial of degree $n$ with constant coefficient $a_{0}$. If $a_{0}$ dominates all the other coefficients of $P$ as in Lemma 5.1, then for all $\mathbf{x} \in[0,1] \times \cdots \times[0,1], P(\mathbf{x})$ has the same sign as $a_{0}$.

Proof. Essentially the same as Lemma 5.1.

Lemma 5.4. Let $\mathbf{x}=\left(x_{1}, \ldots, x_{k}\right)$ and let $P(\mathbf{x})$ be a polynomial of degree $n$. Fix a collection of "stretch values" $t_{1}, \ldots, t_{k}$ and "center values" $p_{1}, \ldots, p_{k}$. Let $Q\left(x_{1}, \ldots, x_{k}\right)=P\left(x_{1} t_{1}+p_{1}, \ldots, x_{k} t_{k}+p_{k}\right)$. Suppose that when $Q$ is expanded out, its constant coefficient $b_{0}$ dominates all its other coefficients as in Lemma 5.1. Then $P(\mathbf{x})$ has the same sign as $b_{0}$ for all $\mathbf{x} \in\left[p_{1}-\right.$ $\left.t_{1}, p_{1}+t_{1}\right] \times \cdots \times\left[p_{k}-t_{k}, p_{k}+t_{k}\right]$.

Proof. The proof follows that of Lemma 5.2.

Now we describe general strategy for proving that a polynomial $P(\mathbf{x})$ is positive on all of a box $\left[a_{1}, b_{1}\right] \times \cdots \times\left[a_{k}, b_{k}\right]$. First, for each $i$ we set $p_{i}=\frac{1}{2}\left(a_{i}+b_{i}\right)$ and $t_{i}=\frac{1}{2}\left(b_{i}-a_{i}\right)$. We test $P$ by Lemma 5.3. If it fails, we divide the box in half in each of the $k$ directions, obtaining $2^{k}$ smaller boxes. We test each of these in turn. The ones that pass, we check off our list, and the ones that still fail, we subdivide in half again. We continue this process until all boxes are small enough to pass, which will eventually be true as long as $P$ actually is strictly positive on all of the original box.

If the coefficients of $P$ and the bounding values $\left\{a_{i}\right\}$ and $\left\{b_{i}\right\}$ of the original box are all rational, then a computer program such as Mathematica can do all of the calculations in exact rational arithmetic, thus eliminating all roundoff error. 


\section{Mathematica programs.}

First we set up the two test functions, Test1Var and Test2Var, as well as the values and functions that will be plugged into them. Test1Var takes a polynomial in one variable with rational coefficients and rational minimum and maximum values prescribed for the domain, and proves (if it succeeds) that the polynomial is positive on the prescribed domain. Similarly, Test2Var does the same for a polynomial in two variables. The main difference in Test2Var is that the domain is usually more complicated to describe.

Line-by-line comments are given below for documenting Test1Var. A few further comments are later added for the features unique to Test2Var.

Note that the lines are numbered along the left side for explanation below. These line numbers are not part of the Mathematica program.

\subsection{The single variable inequality test.}

(1) Test1Var[f, umin, umax $]$ : $=($

(2) $\quad$ blox $=\operatorname{List}[\operatorname{List}[(\operatorname{umin}+\operatorname{umax}) / 2,(\operatorname{umax}-\operatorname{umin}) / 2]]$;

(3) While[Length[blox $]>0$,

(4) For $[\mathrm{i}=1, \mathrm{i}<$ Length $[\mathrm{blox}]+1, \mathrm{i}++$,

(5) $\quad \mathrm{uc}=\operatorname{blox}[[\mathrm{i}, 1]] ; \mathrm{du}=\operatorname{blox}[[\mathrm{i}, 2]]$;

(6) $\quad \operatorname{If}[\mathrm{f}[\mathrm{uc}]<0$, flag $=$ "fail",

(7) If $\left[2^{*} \mathrm{f}[\mathrm{uc}]-\right.$ Apply[Plus, Abs[Flatten[CoefficientList

(8) $\left.\left.\left.\quad\left[\mathrm{f}\left[\mathrm{u}^{*} \mathrm{du}+\mathrm{uc}\right],\{\mathrm{u}\}\right]\right]\right]\right]>0$,

(9) $\quad$ flag $=$ "drop",

(10) flag $=$ "subdivide" 11$]$;

(11) $\quad$ If[flag $==$ "fail", Print [" $\mathrm{f}$ is negative at ", uc, "."]; $\mathrm{i}=2$;

(12) $\quad$ blox $=$ List[]];

(13) If $[$ llag $==$ "drop",

(14) blox $=\operatorname{Join}[$ Take[blox, i - 1], Drop[blox, i]]; i = i - 1];

(15) If $[$ flag $==$ "subdivide",

(16) $\quad \mathrm{hdu}=\mathrm{du} / 2$;

(17) $\quad$ blox $=$ Join[Take[blox, i - 1],

(18) List[List[uc - hdu,hdu],

(19) List[uc + hdu, hdu]],

(20) Drop[blox, i]]; i = i +1$]$;

(21) Print["Number of blocks remaining: ", Length[blox]];

(22) If[Length[blox] > 0, Print["Width: ", N[2*hdu]]]

(23) ] )

\section{Comments:}

Line 1: This program takes as input a polynomial $\mathrm{P}[\mathrm{u}]$, and a closed interval domain (defined by minimum and maximum values of $u$ ) within which to test whether $\mathrm{P}$ is positive. 
Line 2: An individual block (i.e., subinterval) is represented as a list of two numbers, giving its center $u$ value and its radius (half its $u$-width). Line 1 sets up the initial list of "all" blocks, which consists of just one, the original domain.

Line 3: This While statement runs the main For loop over and over until the list of blocks remaining to be checked out is empty.

Line 4: This executes the For loop, which starts with the first block in the list and continues to the last one.

Line 5: Brings out the $i$ th block to be tested.

Lines 6: If the function is negative at the center of the block, then set the flag to report this. Otherwise, go on to Line 7.

Lines 7-9: The command CoefficientList makes a list of the coefficients of the shifted and stretched polynomial $P[u \cdot d u+u c]$. The list has sublist structuring in it, so we Flatten it into a single list, and then take the Absolute value of all the coefficients. The command "Apply[Plus,..." adds these absolute values. The constant coefficient is $P[u c]$, which is nonnegative (because of the test in Line 8), and which is also included in the coefficient list, so that " $2 \mathrm{P}[\mathrm{uc}]-$ Apply[Plus,... " works out to be the constant coefficient minus the sum of absolute values of the remaining coefficients. If this is positive, then the inequality test in Lemma 4 has worked for the current block, so the flag is set to drop the block from the list. Otherwise, go to Line 10.

Line 10: The current block must be subdivided. This line ends the testing of the current block.

Lines 11-12: In this case report test failure and set the values of $i$ and blox so that both the For and the While loops will terminate.

Lines 13-14: In this case remove the current block from the list to be tested, adjust the index i, and return to the For statement to test the next block.

Lines 15-20: In this case subdivide the current block into two blocks of half the size, insert them into the testing list, and adjust the index i so that the reduced blocks aren't tested until the next full pass through the list. Return to the For statement to test the next block in the list.

Lines 21-22: At the end of one full pass of the For statement through all the blocks, report how many blocks remain to be tested and what is the current width of blocks (if there are any). Return to the While statement, which will run the next pass if Lemma 5.3 (i.e., Lines 9-16) has not yet succeeded in eliminating all blocks from the list.

Line 23: This ends the While statement and the definition of Test1Var.

\subsection{The two variable inequality test.}

(1) $g\left[a_{-}\right]:=$Take[a, 2] 
(2) Test2Vars[f, umin, umax, fmin $\left._{-}, \operatorname{vmax}_{-}\right]:=($

(3) blox $=$ List[

(4) $\operatorname{List}[(u \min +u \operatorname{umax}) / 2,(\operatorname{vmin}+\operatorname{vmax}) / 2$,

(5) $\quad(\operatorname{umax}-\operatorname{umin}) / 2,(\operatorname{vmax}-\operatorname{vmin}) / 2]]$;

(6) While[Length[blox $]>0$,

(7) $\operatorname{For}[\mathrm{i}=1, \mathrm{i}<$ Length[blox $]+1, \mathrm{i}++$,

(8) $\quad \mathrm{uc}=\operatorname{blox}[[\mathrm{i}, 1]] ; \mathrm{vc}=\operatorname{blox}[[\mathrm{i}, 2]] ; \mathrm{du}=\operatorname{blox}[[\mathrm{i}, 3]]$;

(9) $\quad \mathrm{dv}=\operatorname{blox}[[\mathrm{i}, 4]]$;

(10) If[blockoutside2 $\operatorname{var}[\mathrm{uc}-\mathrm{du}, \mathrm{uc}+\mathrm{du}$, flag $=$ "drop", $v c-d v, v c+d v$, iter], If [f[uc, vc] $<0 \& \&$ inside2var[uc, vc],

flag = "fail", If $[\mathrm{f}[\mathrm{uc}, \mathrm{vc}]<0$, flag = "subdivide",

If $[2 * f[u c, v c]$ - Apply[Plus, Abs[Flatten[CoefficientList $\left.\left.\left.\left[\mathrm{f}\left[\mathrm{u}^{*} \mathrm{du}+\mathrm{uc}, \mathrm{v}^{*} \mathrm{dv}+\mathrm{vc}\right],\{\mathrm{u}, \mathrm{v}\}\right]\right]\right]\right]>0$, flag $=$ "drop", flag $=$ "subdivide"]]]];

If [flag $==$ "fail", Print ["f is negative at (", uc, ",", vc, ")."]; $\mathrm{i}=2$; blox $=$ List[]]; If $[$ flag $==$ "drop",

blox $=$ Join[Take[blox, i - 1], Drop[blox, i]]; i = i - 1]; If fllag $==$ "subdivide", $\mathrm{hdu}=\mathrm{du} / 2 ; \mathrm{hdv}=\mathrm{dv} / 2$; blox $=\operatorname{Join}[$ Take[blox, i - 1], List[List[uc - hdu, vc - hdv, hdu, hdv], List[uc - hdu, vc + hdv, hdu, hdv], List[uc + hdu, vc - hdv, hdu, hdv], List[uc + hdu, vc + hdv, hdu, hdv]], Drop[blox, i]]; i $=\mathrm{i}+3]$;

If [Length[blox] $>0$, ListPlot[Map[g, blox], PlotRange ->

$\{\{$ umin, umax $\},\{$ vmin, vmax $\}\}]]$; Print["Number of blocks remaining: ", Length[blox]]; (39) ] ) If[Length[blox $]>0$, Print["Width: ", N[2*hdu], " Height: ", N[2*hdv]]]

Comments specific to Test2Var:

1. The function $g$ is for the optional plotting of the centers of the remaining blocks (see Line 35). 
2. We don't want to check positivity of $\mathrm{P}$ on a whole rectangle, but on a specified domain $\mathrm{R}$ inside an initial bounding rectangle. The function blockoutside2var checks whether the current block (sub-rectangle) is completely outside R. If so, the flag is set to drop the block.

3. We only set the flag to "fail" if the block center is actually inside R.

4. If $\mathrm{P}$ is negative and the current block is neither completely outside nor completely inside $\mathrm{R}$, we subdivide.

5. The ListPlot command on Line 34 is optional; it plots all the centers of the blocks not yet dropped, giving a pictorial report of the algorithm's progress.

Side note: This algorithm will not terminate if the function $f$ is exactly equal to zero (but never negative) on part of the domain. In such a case we might analyze derivatives, plugged into the algorithm, in the hope of proving that $f \geq 0$ on the domain.

\section{Inequality tests.}

The purpose of this section is to verify the inequalities (7) and (8) given in the proof of Theorem 4.7. Expressions which convert the associated functions to rational polynomials are defined first. Next, the one variable inequality (7) is proven. Then the shape of the domain of the two variable inequality (8) is described so that it can be determined whether or not a given block meets the domain. Finally, the inequality (8) is verified.

7.1. Defined expressions. We first set up the functions to be tested in the proof of Theorem 4.7. In order to get a polynomial from the trigonometric functions, we start by making the substitutions

$$
\cos \theta=\frac{2 u}{1+u^{2}}, \quad \sin \theta=\frac{1-u^{2}}{1+u^{2}}
$$

and

$$
\cos \left(\frac{\alpha}{2}\right)=\frac{2 v}{1+v^{2}}, \quad \sin \left(\frac{\alpha}{2}\right)=\frac{1-v^{2}}{1+v^{2}} .
$$

Since these are rational functions, rather than polynomials, the functions $N N, n n$ and $R R$ defined below are multiplied by powers of $1+u^{2}$ and of $1+v^{2}$, in a way that does not affect the final inequalities to be proved.

As $u$ ranges from $-\infty$ up to $\infty, \theta$ ranges from $\frac{3 \pi}{2}$ down to $-\frac{\pi}{2}$. We will use $u \in\left[-\frac{3}{2}, \frac{3}{2}\right]$, which corresponds approximately to $\theta \in\left[-\frac{\pi}{8}, \frac{9 \pi}{8}\right]$. To get $\alpha \in\left[0, \frac{\pi}{2}\right]$ we need $0 \leq \frac{\alpha}{2} \leq \frac{\pi}{4}$, which translates to $1 \geq v \geq \sqrt{2}-1$. The value $\sqrt{2}-1$ will be approximated by the slightly smaller value $70 / 169$.

In order to apply the programs to the inequalities in the proof of Theorem 4.7, we define

CosTheta[u_] $:=2 \mathrm{u} /(1+\mathrm{u} \wedge 2)$ 
SinTheta[u_] $:=(1-\mathrm{u} \wedge 2) /(1+\mathrm{u} \wedge 2)$

CosHalfAlpha $\left[\mathrm{v}_{-}\right]:=2 \mathrm{v} /(1+\mathrm{v} \wedge 2)$

SinHalfAlpha[ $\left.\mathrm{v}_{-}\right]:=(1-\mathrm{v} \wedge 2) /(1+\mathrm{v} \wedge 2)$

CosAlpha[ $\left[\mathrm{v}_{-}\right]:=2^{*}$ CosHalfAlpha $[\mathrm{v}] \wedge 2-1$

SinAlpha $\left[\mathrm{v}_{-}\right]:=2^{*}$ SinHalfAlpha $[\mathrm{v}]^{*}$ CosHalfAlpha $[\mathrm{v}]$

$\mathrm{NN}\left[\mathrm{r}_{-}, \mathrm{u}_{-}, \mathrm{v}_{-}\right]:=$List [ Simplify[ Expand[

$\left(\left(\right.\right.$ CosAlpha $[\mathrm{v}]^{*}(1-\mathrm{r} \wedge 2) * \operatorname{Cos}$ Theta $[\mathrm{u}]$

+ SinAlpha $\left.[\mathrm{v}]^{*}(1+\mathrm{r} \wedge 2) * \operatorname{SinTheta}[\mathrm{u}]\right)$

$\left.\left.\left.*\left((1-\mathrm{r} \wedge 2) \wedge 2+\left(2 \mathrm{r}^{*} \operatorname{Cos} \operatorname{Theta}[\mathrm{u}]\right) \wedge 2\right)\right)^{*}(1+\mathrm{u} \wedge 2) \wedge 3^{*}(1+\mathrm{v} \wedge 2) \wedge 2\right]\right]$,

Simplify[ Expand[

((CosAlpha[v]*(1-r^2)*SinTheta[u]

- SinAlpha $[\mathrm{v}]^{*}(1+\mathrm{r} \wedge 2)^{*}$ CosTheta[u] $)$

$\left.\left.\left.\left.*\left((1-\mathrm{r} \wedge 2) \wedge 2+\left(2 \mathrm{r}^{*} \operatorname{SinTheta}[\mathrm{u}]\right) \wedge 2\right)\right)^{*}(1+\mathrm{u} \wedge 2) \wedge 3^{*}(1+\mathrm{v} \wedge 2) \wedge 2\right]\right], 0\right]$

$\mathrm{nn}\left[\mathrm{r}_{-}, \mathrm{u}_{-}, \mathrm{v}_{-}\right]:=\operatorname{List}\left[-2 \mathrm{r}^{*} \operatorname{Cos} \operatorname{Theta}[\mathrm{u}]^{*}(1+\mathrm{v} \wedge 2)^{*}(1+\mathrm{u} \wedge 2)\right.$,

$2 \mathrm{r}^{*}$ SinTheta $\left.[\mathrm{u}]^{*}(1+\mathrm{v} \wedge 2)^{*}(1+\mathrm{u} \wedge 2), 0\right]$

$\mathrm{RR}\left[\mathrm{r}_{-}, \mathrm{u}_{-}, \mathrm{v}_{-}\right]:=\operatorname{List}\left[(1+\mathrm{r} \wedge 2) \operatorname{SinHalfAlpha}[\mathrm{v}]^{*}(1+\mathrm{v} \wedge 2) *(1+\mathrm{u} \wedge 2)\right.$,

$(1+\mathrm{r} \wedge 2)$ CosHalfAlpha[ $\left.\mathrm{v}]^{*}(1+\mathrm{v} \wedge 2) *(1+\mathrm{u} \wedge 2), 0\right]$

fplus $\left[\mathrm{r}_{-}, \mathrm{u}_{-}, \mathrm{v}_{-}\right]:=$Evaluate[Simplify[NN $\left.\left.[\mathrm{r}, \mathrm{u}, \mathrm{v}] \cdot(\mathrm{RR}[\mathrm{r}, \mathrm{u}, \mathrm{v}]+\mathrm{nn}[\mathrm{r}, \mathrm{u}, \mathrm{v}])\right]\right]$ fminus $\left[\mathrm{r}_{-}, \mathrm{u}_{-}, \mathrm{v}_{-}\right]:=$Evaluate[Simplify[NN $\left.\left.[\mathrm{r}, \mathrm{u}, \mathrm{v}] \cdot(\mathrm{RR}[\mathrm{r}, \mathrm{u}, \mathrm{v}]-\mathrm{nn}[\mathrm{r}, \mathrm{u}, \mathrm{v}])\right]\right]$ $G\left[\mathrm{v}_{-}\right]:=-19 / 64+7 / 3 *$ CosHalfAlpha[v] $-4 / 3 *$ CosHalfAlpha[v]^2

- 17/10*SinHalfAlpha[v] + SinHalfAlpha $[\mathrm{v}] \wedge 2$

outsideright2var[u_, $\left.\mathrm{v}_{-}\right]:=\mathrm{If}[\mathrm{G}[\mathrm{v}]<\operatorname{CosTheta}[\mathrm{u}]$, True, False $]$ outsideleft2var[ $\left.\mathrm{u}_{-}, \mathrm{v}_{-}\right]:=\mathrm{If}[\mathrm{G}[\mathrm{v}]>\operatorname{SinTheta}[\mathrm{u}]$

$\& \&$ Not[outsideright2var[u,v]], True, False]

outside2var[ $\left.\mathrm{u}_{-}, \mathrm{v}_{-}\right]:=$outsideright2var[u,v] || outsideleft2var[u,v]

insider $2 \operatorname{var}\left[\mathrm{u}_{-}, \mathrm{v}_{-}\right]:=\operatorname{Not}[$ outside2 $\operatorname{var}[\mathrm{u}, \mathrm{v}]]$

$\mathrm{r} 0=7704 / 10000$

$\mathrm{f} 1\left[\mathrm{u}_{-}, \mathrm{v}_{-}\right]:=$fminus $[\mathrm{r} 0, \mathrm{u}, \mathrm{v}]$

$\mathrm{f} 2\left[\mathrm{u}_{-}, \mathrm{v}_{-}\right]:=$fplus $[\mathrm{r} 0, \mathrm{u}, \mathrm{v}]$

Note that the "Evaluate" command in the definition of fplus and fminus causes the "Simplify" command to occur immediately instead of every time the functions are called.

7.2. Verifying the one variable inequality: Establishing the range for the 2-variable inequality. The following lemma verifies the one variable inequality (7) in the proof of Theorem 4.7: 


\section{Lemma 7.1.}

$$
\tan ^{-1}\left[\frac{1+r^{2}}{1-r^{2}} \tan \alpha\right] \leq \omega(\alpha)
$$

and

$$
\omega(\alpha) \leq \tan ^{-1}\left[\frac{1+r^{2}}{1-r^{2}} \tan \alpha\right]+\frac{\pi}{2}
$$

for $\alpha \in\left[0, \frac{\pi}{2}\right]$, with the inverse tangent defined to equal $\pi / 2$ when $\alpha=\pi / 2$.

Proof. The first inequality is a little delicate to prove near $\alpha=\frac{\pi}{2}$. That is because in order to convert everything to rational functions, we take a cosine and then square both sides. Even though the original inequality is true on the whole interval, there are points near $\frac{\pi}{2}$ for which the squared inequality is false. $\left[0, \frac{\pi}{2}\right]$.

To remedy this, we first note that $\tan ^{-1}\left[\frac{1+r^{2}}{1-r^{2}} \tan \alpha\right] \in\left[0, \frac{\pi}{2}\right]$ for all $\alpha \in$

We will divide $\left[0, \frac{\pi}{2}\right]$ into two intervals $\left[0, a_{0}\right]$ and $\left[a_{0}, \frac{\pi}{2}\right]$, such that the squared inequality is true for $\alpha \in\left[0, a_{0}\right]$, and such that $\omega(\alpha)>\frac{\pi}{2}$ for $\alpha \in$ $\left[a_{0}, \frac{\pi}{2}\right]$. Each of these last inequalities implies the original (1).

Let $a_{0}=\cos ^{-1}\left(\frac{41}{841}\right)$. With the rationalizing substitution $\cos \frac{\alpha}{2}=\frac{2 v}{1+v^{2}}$, $\alpha=a_{0}$ corresponds to $v=3 / 7$. We need to check that $-\cos (\omega(\alpha))>0$ for $\alpha \in\left[a_{0}, \frac{\pi}{2}\right]$, that is, for $v \in[\sqrt{2}-1,3 / 7]$. To get rational endpoints, we will actually prove the inequality for $v \in[2 / 5,3 / 7]$. This is done by the command

$$
\text { Test1Var[Poly2G, 2/5, 3/7], }
$$

where

$$
\begin{aligned}
\text { Poly2G }[v] & =-G[v] *\left(1+v^{2}\right)^{2} \\
& =-\frac{1}{960}\left(-957+4480 v-7610 v^{2}+4480 v^{3}+2307 v^{4}\right) .
\end{aligned}
$$

The test comes back successful.

We now need to prove the "squared inequality" for $\alpha \in\left[0, a_{0}\right]$ :

$$
\begin{gathered}
\tan ^{-1}\left[\frac{1+r^{2}}{1-r^{2}} \tan \alpha\right] \leq \omega(\alpha) \\
\cos \left(\tan ^{-1}\left[\frac{1+r^{2}}{1-r^{2}} \tan \alpha\right]\right) \geq \cos (\omega(\alpha))=G[v] \\
\cos ^{2}\left(\tan ^{-1}\left[\frac{1+r^{2}}{1-r^{2}} \tan \alpha\right]\right) \geq G[v]^{2}
\end{gathered}
$$




$$
\begin{gathered}
\frac{1}{\left[\frac{1+r^{2}}{1-r^{2}} \tan \alpha\right]^{2}+1} \geq G[v]^{2} \\
1 \geq\left(\left[\frac{1+r^{2}}{1-r^{2}} \tan \alpha\right]^{2}+1\right) G[v]^{2} \\
1-\left(\left[\frac{1+r^{2}}{1-r^{2}} \tan \alpha\right]^{2}+1\right) G[v]^{2} \geq 0 \\
1-\left(\left[\frac{1+r^{2}}{1-r^{2}} \frac{4 v\left(v^{2}-1\right)}{v^{4}-6 v^{2}+1}\right]^{2}+1\right) G[v]^{2} \geq 0 .
\end{gathered}
$$

Setting $r=7704 / 10000$ and multiplying both sides by $\left(v^{2}+1\right)^{4}\left(v^{4}-6 v^{2}+1\right)^{2}$ turns the left side into a polynomial which we name MainPoly1[v] and invoke the command

\section{Test1Var[MainPoly1,3/7,1]}

which comes back successful.

To prove inequality (2) above, we first show that $G[v]$ is positive for $v \in[1 / 2,1]$, so that $\cos ^{-1}(\omega(\alpha))<\frac{\pi}{2}$, which implies (2) for the corresponding values of $\alpha$. This is done by defining

Poly $1 \mathrm{G}[v]=G[v] *\left(1+v^{2}\right)^{2}=\frac{1}{960}\left(-957+4480 v-7610 v^{2}+4480 v^{3}+2307 v^{4}\right)$.

We invoke the command

$$
\text { Test1Var[Poly1G,1/2,1] }
$$

which comes back successful.

We then show that for $v \in[2 / 5,1 / 2]$,

$$
G[v] \geq \cos \left(\tan ^{-1}\left[\frac{1+r^{2}}{1-r^{2}} \frac{4 v\left(v^{2}-1\right)}{v^{4}-6 v^{2}+1}\right]+\frac{\pi}{2}\right) .
$$

Here the right side is negative, so the above inequality would follow from

$$
\begin{aligned}
G[v]^{2} & \leq \cos ^{2}\left(\tan ^{-1}\left[\frac{1+r^{2}}{1-r^{2}} \frac{4 v\left(v^{2}-1\right)}{v^{4}-6 v^{2}+1}\right]+\frac{\pi}{2}\right) \\
& =\sin ^{2}\left(\tan ^{-1}\left[\frac{\left(1+r^{2}\right)(4 v)\left(v^{2}-1\right)}{\left(1-r^{2}\right)\left(v^{4}-6 v^{2}+1\right)}\right]\right) \\
& =\frac{\left[\frac{\left(1+r^{2}\right)(4 v)\left(v^{2}-1\right)}{\left(1-r^{2}\right)\left(v^{4}-6 v^{2}+1\right)}\right]^{2}}{\left[\frac{\left(1+r^{2}\right)(4 v)\left(v^{2}-1\right)}{\left(1-r^{2}\right)\left(v^{4}-6 v^{2}+1\right)}\right]^{2}+1} .
\end{aligned}
$$


Hence

$$
\begin{gathered}
\left(\left[\frac{\left(1+r^{2}\right)(4 v)\left(v^{2}-1\right)}{\left(1-r^{2}\right)\left(v^{4}-6 v^{2}+1\right)}\right]^{2}+1\right) G[v]^{2} \leq\left[\frac{\left(1+r^{2}\right)(4 v)\left(v^{2}-1\right)}{\left(1-r^{2}\right)\left(v^{4}-6 v^{2}+1\right)}\right]^{2}, \\
\left(\left[\left(1+r^{2}\right)(4 v)\left(v^{2}-1\right)\right]^{2}+\left[\left(1-r^{2}\right)\left(v^{4}-6 v^{2}+1\right)\right]^{2}\right) G[v]^{2} \\
\leq\left[\left(1+r^{2}\right)(4 v)\left(v^{2}-1\right)\right]^{2},
\end{gathered}
$$

and

$$
\begin{aligned}
{\left[\left(1+r^{2}\right)(4 v)\left(v^{2}-1\right)\right]^{2}-([(1+} & \left.\left.r^{2}\right)(4 v)\left(v^{2}-1\right)\right]^{2} \\
+ & {\left.\left[\left(1-r^{2}\right)\left(v^{4}-6 v^{2}+1\right)\right]^{2}\right) G[v]^{2} \geq 0 . }
\end{aligned}
$$

Multiplying by $\left(1+v^{2}\right)^{4}$ to make the above a polynomial, we test it for $v \in[2 / 5,1 / 2]$. Test1Var immediately confirms to be positive.

7.3. Establishing the shape of the domain. Now to justify the test called "blockoutside2var" in the program "Test2Var," we need to show that if the two right-hand corners of a rectangle are to the left of $\mathrm{R}$, or if the two left-hand corners of the rectangle are to the right of $\mathrm{R}$, then the whole rectangle is outside $\mathrm{R}$. The top and bottom of $\mathrm{R}$ are the straight lines $v=\frac{70}{169}$ and $v=1$, and the top and bottom of a subrectangle will always be in $\left[\frac{70}{169}, 1\right]$. We will show that the left and right sides of $\mathrm{R}$ are formed by functions $u_{1}(v)<u_{2}(v)$ which are both increasing functions, from which the desired result will follow. We start with the following lemma:

Lemma 7.2. The function $G[v]$ is increasing on $[70 / 169,1]$.

Proof. We take the first derivative $G^{\prime}(v)$ and multiply it by $\left(1+v^{2}\right)^{3}$, obtaining the polynomial

$$
\frac{14}{3}-\frac{178}{15} v+\frac{382}{15} v^{3}-\frac{14}{3} v^{4}
$$

To show this is positive on $[70 / 169,1]$, we call it $G P[v]$ and invoke the command

$$
\text { Test1Var[GP,70/169,1] }
$$

which is successful.

Lemma 7.3. Within the rectangle $(u, v) \in\left[-\frac{3}{2} \frac{3}{2}\right] \times\left[\frac{70}{169}, 1\right]$, the expressions

$$
\frac{1-u^{2}}{1+u^{2}}=G(v) \geq \frac{2 u}{1+u^{2}}
$$

and

$$
\frac{2 u}{1+u^{2}}=G(v)
$$


implicitly define single-valued, increasing functions $u=u_{1}(v)$ and $u=u_{2}(v)$, respectively, with $u_{1}(v)<u_{2}(v)$.

Proof. First, calculations show that $G\left[\frac{70}{169}\right]>-0.016$ and $G[1]<0.704$. Since $G$ is increasing, its values on all of $\left[\frac{70}{169}, 1\right]$ are between these endpoint values.

Now it is straightforward to show that $2 u /\left(1+u^{2}\right)>0.9$ on $[3 / 4,3 / 2]$ and $2 u /\left(1+u^{2}\right)<-0.4$ on $[-3 / 2,-1 / 4]$. So, given the range of $G[v]$, Equation (2) will only allow values of $u$ within $[-1 / 4,3 / 4]$. On this interval, the function $2 u /\left(1+u^{2}\right)$ is strictly increasing. Taking these facts together, we see that there will be exactly one $u$ for each $v \in\left[\frac{70}{169}, 1\right]$ for which (2) holds, and that the function $u=u_{2}(v)$ thereby defined is a strictly increasing function.

Next, within $[-3 / 2,3 / 2]$ the inequality $\left(1-u^{2}\right) /\left(1+u^{2}\right) \geq 2 u /\left(1+u^{2}\right)$ is true precisely for $u \in[-3 / 2, \sqrt{2}-1]$. But for $u \in[-(\sqrt{2}-1), \sqrt{2}-1]$, $\left(1-u^{2}\right) /\left(1+u^{2}\right) \geq \sqrt{2} / 2$, and so $\left(1-u^{2}\right) /\left(1+u^{2}\right)$ is outside the range of $G[v]$. Thus, for (1) to hold, $u$ must be within $[-3 / 2,1-\sqrt{2}]$. On this interval, $\left(1-u^{2}\right) /\left(1+u^{2}\right)$ is strictly increasing, and again we obtain a single-valued, increasing function $u=u_{1}(v)$ implicitly defined by (1).

Finally, since $-1 / 4 \leq u_{2} \leq 3 / 4$ and $-3 / 2 \leq u_{1} \leq 1-\sqrt{2}<-1 / 4$, we see that $u_{1}(v)<u_{2}(v)$.

From the above lemma, we infer that the shape of $\mathrm{R}$ is such that the following proposition holds:

Propositon 7.4. If the $v$-values at the top and bottom of a rectangle $B$ are within $[70 / 169,1]$, and if either the two right corners of $B$ are to the right of $R$ or the two left corners of $B$ are to the right of $R$, then the entire rectangle is outside $R$.

Finally, we use the following lemma to justify the tests "outsideright2var" and "outsideleft2var". In the lemma $K$ represents $G[v]$ which does lie between $-\frac{\sqrt{2}}{2}$ and $\frac{\sqrt{2}}{2}$, as noted previously.

Lemma 7.5. If

$$
-\frac{\sqrt{2}}{2} \leq K \leq \frac{\sqrt{2}}{2}
$$

and

then

$$
\cos ^{-1} K \leq \theta \leq \cos ^{-1} K+\frac{\pi}{2}
$$

$$
\cos \theta \leq K \leq \sin \theta
$$

Proof. First note that $\frac{\pi}{4} \leq \cos ^{-1} K \leq \frac{3 \pi}{4}$, and thus $\frac{\pi}{4} \leq \theta \leq \frac{5 \pi}{4}$. In particular, $\cos ^{-1} K \in[0, \pi]$, where the cosine function is decreasing. To prove that $\cos \theta \leq K$, consider 
Case a1: $\theta \in[0, \pi]$. The fact that $\cos ^{-1} K \leq \theta$ implies $K=\cos \left(\cos ^{-1} K\right)$ $\geq \cos \theta$.

Case a2: $\theta \in\left[\pi, \frac{5 \pi}{4}\right]$. Then $\cos \theta \leq-\frac{\sqrt{2}}{2} \leq K$.

Now to prove that $K \leq \sin \theta$, consider:

Case b1: $\theta \in\left[\frac{\pi}{4}, \frac{3 \pi}{4}\right]$. Then $K \leq \frac{\sqrt{2}}{2} \leq \sin \theta$.

Case b2: $\theta \in\left[\frac{3 \pi}{4}, \frac{5 \pi}{4}\right]$. Then both $\theta$ and $\cos ^{-1} K+\frac{\pi}{2}$ are in an interval where the sine function is decreasing. So the fact that $\theta \leq \cos ^{-1} K+\frac{\pi}{2}$ implies that $\sin \theta \geq \sin \left(\cos ^{-1} K+\frac{\pi}{2}\right)=K$, as desired.

7.4. The 2-variable inequality. The polynomials $f 1(u, v)$ and $f 2(u, v)$ are the functions we need to prove positive on $\sqrt{2}-1 \leq v \leq 1, G(v) \leq u \leq$ $G(v)+\frac{\pi}{2}$ in order to verify the inequality (8) in the proof of Theorem 4.7 . The following two commands accomplish this:

$$
\begin{aligned}
& \text { Test2Vars[f1, }-3 / 2,3 / 2,70 / 169,1] \\
& \text { Test2Vars[f2, -3/2, 3/2, 70/169, 1]. }
\end{aligned}
$$

The test comes back positive.

\section{References}

[1] J. Clunie and T. Sheil-Small, Harmonic univalent functions, Ann. Acad. Sci. Fenn. Ser. A.I Math., 9 (1984), 3-25, MR 85i:30014, Zbl 0539.30006.

[2] U. Dierkes, S. Hildebrandt, A. Küster and O. Wohlrab, Minimal Surfaces I, SpringerVerlag, Belin-Heidelberg-New York, 1992, MR 94c:49001a, Zbl 0777.53012.

[3] M. Dorff, Minimal graphs in $\mathbb{R}^{3}$ over convex domains, to appear in Proc. Amer. Math. Soc.

[4] P. Duren, A survey of harmonic mappings in the plane, Texas Tech. Univ., Math. Series, Visiting Scholars Lectures, 1990-1992, 18 (1992), 1-15.

[5] _ Univalent Functions, Springer-Verlag, New York, 1983, MR 85j:30034, Zbl 0514.30001.

[6] H. Federer, Geometric Measure Theory, Springer-Verlag, New York, 1969, MR 41 \#1976, Zbl 0176.00801.

[7] H. Karcher, Construction of Minimal Surfaces, Lecture Notes, 12, SFB256, Bonn, 1989.

[8] W. Koepf, On close-to-convex functions and linearly accessible domains, Complex Variables Theory Appl., 11(3-4) (1989), 269-279, MR 91f:30010, Zbl 0679.30007.

[9] G. Lawlor, Proving area minimization by directed slicing, Indiana Univ. Math. J., 47 (1998), 1547-1592, MR 2001b:49057, Zbl 0944.49030.

[10] Z. Lewandowski, Sur l'indentitè de certaines classes de fonctions univalentes I, Ann. Univ. Mariae Curie-Sklodowska, Sect. A, 12 (1958), 131-146, MR 24 \#A217, Zbl 0108.07502.

[11] F. Morgan, Geometric Measure Theory: A Beginner's Guide, 2nd edition, Academic Press, San Diego, 1995, MR 96c:49001, Zbl 0819.49024. 
[12] J.C.C. Nitsche, Non-uniqueness for Plateau's problem: A bifurcation process, Ann. Acad. Sci. Fenn. Ser. A I Math., 2 (1976), 361-373, MR 57 \#7408, Zbl 0361.49025.

[13] J. Oprea, The Mathematics of Soap Films: Explorations with Maple, Student Mathematical Library, 10, AMS, MR 2002c:53013, Zbl 0972.53003.

[14] H. Ruchert, A uniqueness result for Enneper's minimal surface, Indiana Univ. Math. J., 30(3) (1981), 427-431, MR 82h:53012, Zbl 0492.49022.

[15] B. White, Half of Enneper's surface minimizes area, Geometric Analysis and the Calculus of Variations, 361-367, Internat. Press, Cambridge, MA, 1996, MR 98c:51003.

Received April 4, 2002.

Department of Mathematics

Brigham Young University

Provo, Utah 84602

E-mail address: mdorff@math.byu.edu

Department of Mathematics

Brigham Young University

Provo, Utah 84602

E-mail address: deniseh@math.byu.edu

Department of Mathematics

Brigham Young UnIVERSity

Provo, Utah 84602

E-mail address: lawlor@mathed.byu.edu 